\title{
A New Similarity Measure of Interval-Valued Intuitionistic Fuzzy Sets Considering Its Hesitancy Degree and Applications in Expert Systems
}

\author{
Chong Wu, ${ }^{1}$ Peng Luo, ${ }^{1}$ Yongli Li, ${ }^{1}$ and Xuekun Ren ${ }^{2}$ \\ ${ }^{1}$ School of Management, Harbin Institute of Technology, West Street 92, Harbin 150001, China \\ ${ }^{2}$ Department of Mathematics, Harbin Institute of Technology, Harbin 150001, China \\ Correspondence should be addressed to Peng Luo; luopeng_hit@126.com
}

Received 9 December 2013; Revised 2 March 2014; Accepted 2 March 2014; Published 11 May 2014

Academic Editor: Bo Shen

Copyright (C) 2014 Chong Wu et al. This is an open access article distributed under the Creative Commons Attribution License, which permits unrestricted use, distribution, and reproduction in any medium, provided the original work is properly cited.

As an important content in fuzzy mathematics, similarity measure is used to measure the similarity degree between two fuzzy sets. Considering the existing similarity measures, most of them do not consider the hesitancy degree and some methods considering the hesitancy degree are based on the intuitionistic fuzzy sets, intuitionistic fuzzy values. It may cause some counterintuitive results in some cases. In order to make up for the drawback, we present a new approach to construct the similarity measure between two interval-valued intuitionistic fuzzy sets using the entropy measure and considering the hesitancy degree. In particular, the proposed measure was demonstrated to yield a similarity measure. Besides, some examples are given to prove the practicality and effectiveness of the new measure. We also apply the similarity measure to expert system to solve the problems on pattern recognition and the multicriteria group decision making. In these examples, we also compare it with existing methods such as other similarity measures and the ideal point method.

\section{Introduction}

In 1989, interval-valued intuitionistic fuzzy set (IVIFS), which is characterized by a membership degree range and a nonmembership degree range, was proposed by Atanassov and Gargov [1]. Due to the high complexity of the real world and the limitation of people's judgment, the use of interval-valued intuitionistic fuzzy set has gained much attention [2-9]. As one of the most important applications, the expert system to solve problems under fuzzy environment is a hot research topic [10-15]. We give an example of the decision problem which is also an important aspect of the expert system. As the time pressure and lack of data, the decision information is often imprecise or uncertain. The experts may not express their preference over the alternatives considered precisely. So the evaluation can be given in the form of interval-valued intuitionistic fuzzy numbers [1]. Accordingly, IVIFS is a very suitable tool to describe the imprecise or uncertain decision information and deal with the uncertainty and vagueness in decision making.

As one of the important topics of fuzzy theory, the similarity measures are used to estimate the degree of similarity between two fuzzy sets. Functions expressing the degree of similarity of items or sets are used in many different fields, such as numerical taxonomy, ecology, information retrieval, and psychology and the similarity measure plays a very important role [16]. For example, many scholars take advantages of the similarity measures, especially the Jaccard, Dice, and cosine methods [17-19] to research the problems in information retrieval, citation analysis, and automatic classification. Therefore, the similarity measure has been investigated by many authors [20-22]. For the similarity measure to IVIFSs, $\mathrm{Xu}$ and Chen [23] generalized some formulas of similarity measures which are based on the distance measures for two IVIFSs. Besides, Wei et al. [24] gave a formula of a similarity measure of IVIFSs based on entropy 
theory [25]. Ye [16] presented a Dice similarity measure based on the reduct intuitionistic fuzzy sets of interval-valued intuitionistic fuzzy sets and applied it to multicriteria decision making problems. Singh [26] and Ye [21] also proposed a new cosine similarity measure for two IVIFSs, respectively. $\mathrm{Xu}$ and Yager [27] had studied the intuitionistic and intervalvalued intuitionistic fuzzy preference relations and suggested some new similarity measures to evaluate the agreement within a group. However, many of these measures for two IVIFSs do not consider the degree of hesitancy and may lead to counterintuitive results in some cases. For example, for $A, B, C \in$ IVIFSs, we assume that the differences of membership and nonmembership degree in $A, B$ and $A, C$ are very close and the difference of hesitancy degree between $B$ and $C$ is very large. Then, it may be incapable of distinguishing which one between $B$ and $C$ is more similar to $A$ using the existing similarity measure. Besides, many other similarity measures for intuitionistic fuzzy values or intuitionistic fuzzy sets have been proposed. $\mathrm{Xu}$ [28] developed some similarity measures of intuitionistic fuzzy sets which consider the hesitancy degree and also define the notions of positive ideal intuitionistic fuzzy set and negative ideal intuitionistic fuzzy set. Later, Xia and Xu [29] proposed a series of similarity measures for intuitionistic fuzzy values (IFVs) based on the intuitionistic fuzzy operators and the similarity measures were also taking the hesitancy degree into consideration. In 2011, Xu and Xia [30] also studied the distance and similarity measures for hesitant fuzzy sets. The similarity measures considering the hesitancy degree outperform the existing methods and can deal with the group decision well. So we establish a new method of similarity measure by taking the hesitancy degree into consideration and using the entropy measure of IVIFSs.

The structure of this paper is organized as follows. Section 2 focuses on the concepts of the interval-valued intuitionistic fuzzy sets and analyses the drawbacks of some existing similarity measures. Section 3 presents a new similarity measure and illustrates its advantage. Section 4 describes the application to expert system based on the similarity measure of the IVIFSs and presents some illustrative examples. Section 5 concludes our work.

\section{Basic Notions and the Drawback of Existing Similarity Measures}

\subsection{Preliminaries}

Definition 1 (see [1]). Aninterval-valued intuitionistic fuzzy set in $X$ is an expression $A$ given by

$$
A=\left\{\left\langle x, u_{A}(x), v_{A}(x)\right\rangle \mid x \in X\right\},
$$

where

$$
u_{A}: X \longrightarrow[0,1], \quad v_{A}: X \longrightarrow[0,1]
$$

with the condition $0 \leq u_{A}(x)+v_{A}(x) \leq 1$ for all $x \in X$. The intervals $u_{A}(x)$ and $v_{A}(x)$ denote the degree of membership and nonmembership of $x$ to $A$, respectively.
If we let $u_{A}(x)=\left[u_{A}^{-}(x), u_{A}^{+}(x)\right], v_{A}(x)=\left[v_{A}^{-}(x), v_{A}^{+}(x)\right]$, the interval-valued intuitionistic fuzzy set $A$ can be represented as follows:

$$
A=\left\{\left\langle x,\left[u_{A}^{-}(x), u_{A}^{+}(x)\right],\left[v_{A}^{-}(x), v_{A}^{+}(x)\right]\right\rangle \mid x \in X\right\},
$$

where $0 \leq u_{A}^{+}(x)+v_{A}^{+}(x) \leq 1$. The interval,

$$
\left[1-u_{A}^{+}(x)-v_{A}^{+}(x), 1-u_{A}^{-}(x)-v_{A}^{-}(x)\right],
$$

is denoted by $\pi_{A}$ and abbreviated by $\left[\pi_{A}^{-}, \pi_{A}^{+}\right]$, which is called the intuitionistic index of element $x$ in $A$. We call it a hesitancy degree of $x$ to $A$.

For all $A, B \in \operatorname{IVFSs}(X)$, we have the following expression defined in [1]:

(1) $A \subseteq B$ if and only if $u_{A}^{-}(x) \leq u_{B}^{-}(x), u_{A}^{+}(x) \leq u_{B}^{+}(x)$, $v_{A}^{-}(x) \geq v_{B}^{-}(x), v_{A}^{+}(x) \geq v_{B}^{+}(x)$ for $x \in X ;$

(2) $A=B$ if and only if $A \subseteq B, A \supseteq B$;

(3) $A^{c}=\left\{\left(x,\left[v_{A}^{-}(x), v_{A}^{+}(x)\right],\left[u_{A}^{-}(x), u_{A}^{+}(x)\right]\right) \mid x \in X\right\}$.

Definition 2 (see [24]). For $A \in \operatorname{IVFSs}(X)$, an entropy measure on $\operatorname{IVFSs}(X)$ can be given by

$$
\begin{aligned}
E(A)=\frac{1}{n} \sum_{j=1}^{n}( & \min \left\{u_{A}^{-}\left(x_{j}\right), v_{A}^{-}\left(x_{j}\right)\right\} \\
& +\min \left\{u_{A}^{+}\left(x_{j}\right), v_{A}^{+}\left(x_{j}\right)\right\} \\
& \left.+\pi_{A}^{-}\left(x_{j}\right)+\pi_{A}^{+}\left(x_{j}\right)\right) \\
\times & \left(\max \left\{u_{A}^{-}\left(x_{j}\right), v_{A}^{-}\left(x_{j}\right)\right\}\right. \\
& +\max \left\{u_{A}^{+}\left(x_{j}\right), v_{A}^{+}\left(x_{j}\right)\right\} \\
& \left.+\pi_{A}^{-}\left(x_{j}\right)+\pi_{A}^{+}\left(x_{j}\right)\right)^{-1} .
\end{aligned}
$$

It also has the following properties.

(1) $E(A)=0$ if and only if $A$ is the crispest.

(2) $E(A)=1$ if and only if $\left[u_{A}^{-}\left(x_{j}\right), u_{A}^{+}\left(x_{j}\right)\right]=$ $\left[v_{A}^{-}\left(x_{j}\right), v_{A}^{+}\left(x_{j}\right)\right]$ for all $x_{j} \in X$.

(3) $E(A)=E\left(A^{c}\right)$.

(4) $E(A) \leq E(B)$ if $A \subseteq B$ when $u_{B}^{+}\left(x_{j}\right) \leq v_{B}^{+}\left(x_{j}\right)$ and $u_{B}^{-}\left(x_{j}\right) \leq v_{B}^{-}\left(x_{j}\right)$, for $x_{j} \in X$, or $A \supseteq B$ when $u_{B}^{+}\left(x_{j}\right) \geq$ $v_{B}^{+}\left(x_{j}\right)$ and $u_{B}^{-}\left(x_{j}\right) \geq v_{B}^{-}\left(x_{j}\right)$ for $x_{j} \in X$.

Definition 3 (see [21]). Let $A, B \in \operatorname{IVFS}(X), S: \operatorname{IVFS}(X) \times$ $\operatorname{IVFS}(X) \rightarrow[0,1]$; then, the degree of similarity between $A$ and $B$ is $S(A, B)$, if it satisfies the following requirements:

(1) $0 \leq S(A, B) \leq 1$;

(2) $S(A, B)=1$ if and only if $A=B$;

(3) $S(A, B)=S(B, A)$;

(4) if $A, B, C \in \operatorname{IVFS}(X), A \subseteq B \subseteq C$, then $S(A, C) \leq$ $S(A, B), S(A, C) \leq S(B, C)$. 
2.2. The Existing Similarity Measures. Wei et al. [24] define a method to construct similarity measure of IVFSs as follows. Firstly, for $A, B \in \operatorname{IVFSs}(X)$, a new IVFS is defined, written by $M(A, B)$. Consider

$$
\begin{aligned}
& M_{A B 1}(x)=\frac{1+\min \left\{\left|u_{A}^{-}(x)-u_{B}^{-}(x)\right|,\left|v_{A}^{-}(x)-v_{B}^{-}(x)\right|\right\}}{2}, \\
& M_{A B 2}(x)=\frac{1+\min \left\{\left|u_{A}^{+}(x)-u_{B}^{+}(x)\right|,\left|v_{A}^{+}(x)-v_{B}^{+}(x)\right|\right\}}{2}, \\
& M_{A B 3}(x)=\frac{1-\max \left\{\left|u_{A}^{-}(x)-u_{B}^{-}(x)\right|,\left|v_{A}^{-}(x)-v_{B}^{-}(x)\right|\right\}}{2}, \\
& M_{A B 4}(x)=\frac{1-\max \left\{\left|u_{A}^{+}(x)-u_{B}^{+}(x)\right|,\left|v_{A}^{+}(x)-v_{B}^{+}(x)\right|\right\}}{2} .
\end{aligned}
$$

Let

$$
\begin{aligned}
& u_{M(A, B)}^{-}(x)=\min \left\{M_{A B 1}(x), M_{A B 2}(x)\right\}, \\
& u_{M(A, B)}^{+}(x)=\max \left\{M_{A B 1}(x), M_{A B 2}(x)\right\}, \\
& v_{M(A, B)}^{-}(x)=\min \left\{M_{A B 3}(x), M_{A B 4}(x)\right\}, \\
& v_{M(A, B)}^{+}(x)=\max \left\{M_{A B 3}(x), M_{A B 4}(x)\right\} .
\end{aligned}
$$

Then we get

$$
\begin{aligned}
M(A, B)=\{\langle & {\left[u_{M(A, B)}^{-}(x), u_{M(A, B)}^{+}(x)\right], } \\
& {\left.\left.\left[v_{M(A, B)}^{-}(x), v_{M(A, B)}^{+}(x)\right]\right\rangle \mid x \in X\right\} . }
\end{aligned}
$$

Then $M(A, B) \in \operatorname{IVFSs}(X)$ and the degree of similarity between $A$ and $B$ is $S_{1}(A, B)=E(M(A, B))$.

$\mathrm{Xu}$ [31] also defines four different similarity measures of $A$ and $B$. We assume that $X$ is finite; that is, $X=\left\{x_{1}, x_{2}, \ldots, x_{n}\right\}$ and $j=\{1,2, \ldots, n\}$. Let

$$
\begin{array}{r}
A=\left\{\left(x_{j},\left[u_{A}^{-}\left(x_{j}\right), u_{A}^{+}\left(x_{j}\right)\right],\right.\right. \\
\left.\left.\left[v_{A}^{-}\left(x_{j}\right), v_{A}^{+}\left(x_{j}\right)\right]\right) \mid x_{j} \in X\right\}, \\
B=\left\{\left(x_{j},\left[u_{B}^{-}\left(x_{j}\right), u_{B}^{+}\left(x_{j}\right)\right],\right.\right. \\
\left.\left.\left[v_{B}^{-}\left(x_{j}\right), v_{B}^{+}\left(x_{j}\right)\right]\right) \mid x_{j} \in X\right\}
\end{array}
$$

be two IVFSs. Let $w=\left\{w_{1}, w_{2}, \ldots, w_{n}\right\}$ be the weight vector of elements $x_{j}, j=1,2, \ldots, n$. The similarity measures of $A$ and $B$ are as follows:

$S_{2}(A, B)$

$$
\begin{aligned}
=1-\frac{1}{4} \sum_{j=1}^{n} w_{j}\left[\left|u_{A}^{-}\left(x_{j}\right)-u_{B}^{-}\left(x_{j}\right)\right|+\left|u_{A}^{+}\left(x_{j}\right)-u_{B}^{+}\left(x_{j}\right)\right|\right. \\
+\left|v_{A}^{-}\left(x_{j}\right)-v_{B}^{-}\left(x_{j}\right)\right| \\
\left.+\left|v_{A}^{+}\left(x_{j}\right)-v_{B}^{+}\left(x_{j}\right)\right|\right]
\end{aligned}
$$

$S_{3}(A, B)$

$$
\begin{aligned}
=1-\left(\frac{1}{4} \sum_{j=1}^{n} w_{j}[\right. & \left(u_{A}^{-}\left(x_{j}\right)-u_{B}^{-}\left(x_{j}\right)\right)^{2} \\
& +\left(u_{A}^{+}\left(x_{j}\right)-u_{B}^{+}\left(x_{j}\right)\right)^{2} \\
& +\left(v_{A}^{-}\left(x_{j}\right)-v_{B}^{-}\left(x_{j}\right)\right)^{2} \\
& \left.\left.+\left(v_{A}^{+}\left(x_{j}\right)-v_{B}^{+}\left(x_{j}\right)\right)^{2}\right]\right)^{1 / 2},
\end{aligned}
$$

$S_{4}(A, B)$

$$
\begin{aligned}
& =1-\sum_{j=1}^{n} w_{j} \\
& \times \max \left\{\left|u_{A}^{-}\left(x_{j}\right)-u_{B}^{-}\left(x_{j}\right)\right|,\left|u_{A}^{+}\left(x_{j}\right)-u_{B}^{+}\left(x_{j}\right)\right|,\right. \\
& \left.\left|v_{A}^{-}\left(x_{j}\right)-v_{B}^{-}\left(x_{j}\right)\right|,\left|v_{A}^{+}\left(x_{j}\right)-v_{B}^{+}\left(x_{j}\right)\right|\right\},
\end{aligned}
$$

$S_{5}(A, B)$

$$
\begin{aligned}
=1-\left(\sum_{j=1}^{n} w_{j} \max \{\right. & \left(u_{A}^{-}\left(x_{j}\right)-u_{B}^{-}\left(x_{j}\right)\right)^{2}, \\
& \left(u_{A}^{+}\left(x_{j}\right)-u_{B}^{+}\left(x_{j}\right)\right)^{2}, \\
& \left(v_{A}^{-}\left(x_{j}\right)-v_{B}^{-}\left(x_{j}\right)\right)^{2}, \\
& \left.\left.\left(v_{A}^{+}\left(x_{j}\right)-v_{B}^{+}\left(x_{j}\right)\right)^{2}\right\}\right)^{1 / 2} .
\end{aligned}
$$

2.3. The Drawback of Existing Similarity Measures. We give an example to calculate the similarity of two fuzzy sets using the above five different similarity measures. It proves that the five different similarity measures are not reliable in some cases. 
Example 4. It is an example of group voting. We have three plans written in the form of IFSs. Let $A=\{x, 0.5,0.5\}, B=$ $\{x, 0.3,0.7\}$, and $C=\{x, 0.3,0.3\}$ be three IFSs and denote the three plans. Plan $A$ denotes that the percentage of supporters or dissenters is fifty percent. As to plan $B$, thirty percent of the voters support the plan and seventy percent of the voters oppose it. For plan $C$, we can know that the percentage of supporters or dissenters is thirty percent while forty percent of the voters abstain from voting.

Then we can get the results of the similarity of $A$ and $B$ based on the above similarity measure. Consider

$$
\begin{aligned}
& M_{A B 1}(x) \\
& \quad=M_{A B 2}(x) \\
& \quad=0.5 *\left(1+\min \left\{\left|u_{A}(x)-u_{B}(x)\right|,\left|v_{A}(x)-v_{B}(x)\right|\right\}\right) \\
& \quad=0.6, \\
& M_{A B 3}(x) \\
& \quad=M_{A B 4}(x) \\
& \quad=0.5 *\left(1-\max \left\{\left|u_{A}(x)-u_{B}(x)\right|,\left|v_{A}(x)-v_{B}(x)\right|\right\}\right) \\
& \quad=0.4, \\
& M(A, B)=\{x, 0.6,0.4\}, \quad S_{1}(A, B)=E(M(A, B))=\frac{2}{3} .
\end{aligned}
$$

In the same way, we can get the similarity of $A$ and $C$; that is, $S_{1}(A, C)=E(M(A, C))=2 / 3$. Supposing $w_{j}=1, n=1$, we can get the following results:

$$
\begin{aligned}
S_{2}(A, B) & =S_{2}(A, C) \\
& =1-0.25 *[0.2+0.2+0.2+0.2]=0.8, \\
S_{3}(A, B) & =S_{3}(A, C) \\
& =1-\sqrt{0.25 *[0.04+0.04+0.04+0.04]}=0.8, \\
S_{4}(A, B) & =S_{4}(A, C)=1-\sum \max \{0.2,0.2,0.2,0.2\}=0.8, \\
S_{5}(A, B) & =S_{5}(A, C) \\
& =1-\sqrt{\sum \max \{0.04,0.04,0.04,0.04\}}=0.8 .
\end{aligned}
$$

Obviously, we could not distinguish which one between $B$ and $C$ is more similar to $A$ using the five different similarity measures. However, if we make a deep analysis, we can know that $A$ is more similar to $C$ than to $B$. According to formula (4), the hesitancy degree of $C$ is twenty percent while the hesitancy degree of $B$ is zero. For plan $C$, the reason of abstention may be different. For example, someone may be absent for the vote. So, it is reasonable to assume that the twenty percent of abstention has a certain degree $r(0 \leq r \leq$ 1) to support the plan.
If $r=0$ or $r=1$, all of the abstainers oppose or support the plan $C$. We have the following results:

$$
\begin{gathered}
C=\{x, 0.3,0.7\} \quad \text { or } \quad C=\{x, 0.7,0.3\} \\
S_{i}(A, C)=S_{i}(A, B), \quad i=1,2, \ldots, 5
\end{gathered}
$$

If $r=0.5$, that is, half of the abstainers to plan $C$ are supporters or dissenters, the percentage of supporters or dissenters is fifty percent. We can get the result as follows:

$$
\begin{array}{cc}
C=\{x, 0.5,0.5\}, & S_{i}(A, C)=1, \\
S_{i}(A, C)>S_{i}(A, B), & i=1,2, \ldots, 5 .
\end{array}
$$

But in general, both situations do not always happen, and it is just between them; that is, $0<r<0.5,0.5<r<1$. So we can get the result as follows: $S(A, C)>S(A, B)$.

According to our analysis, more reasonable result is that $A$ is more similar to $C$ than to $B$. It is different from the result calculated by the existing five similarity measures. Besides, we can also know that the five similarity measures do not take the hesitancy degree into consideration according to its definition. It is not reasonable to define the similarity measure without considering the hesitancy degree of the interval-valued intuitionistic fuzzy sets; otherwise, it may make mistakes in some cases. So we present a new method of similarity measure.

\section{The New Similarity Measures between IVIFSs}

3.1. New Similarity Measure. Let $X=\left\{x_{1}, x_{2}, \ldots, x_{n}\right\}$ be a finite universe of discourse. For $A, B \in \operatorname{IVFSs}(X)$, we define IVFSs, written by $M(A, B)$ :

$$
\begin{aligned}
M_{A B 1}\left(x_{j}\right)=(2+ & \left|u_{A}^{-}\left(x_{j}\right)-u_{B}^{-}\left(x_{j}\right)\right| \\
& +\left|v_{A}^{-}\left(x_{j}\right)-v_{B}^{-}\left(x_{j}\right)\right| \\
& \left.-\left(\pi_{A}^{+}\left(x_{j}\right)+\pi_{B}^{+}\left(x_{j}\right)\right)\right) \times 4^{-1}, \\
M_{A B 2}\left(x_{j}\right)=(2+ & \left|u_{A}^{+}\left(x_{j}\right)-u_{B}^{+}\left(x_{j}\right)\right| \\
& +\left|v_{A}^{+}\left(x_{j}\right)-v_{B}^{+}\left(x_{j}\right)\right| \\
& \left.-\left(\pi_{A}^{-}\left(x_{j}\right)+\pi_{B}^{-}\left(x_{j}\right)\right)\right) \times 4^{-1}, \\
& -\left(\left|u_{A}^{-}\left(x_{j}\right)-u_{B}^{-}\left(x_{j}\right)\right|\right. \\
M_{A B 3}\left(x_{j}\right)=\left(2-v_{A}^{-}\left(x_{j}\right)-v_{B}^{-}\left(x_{j}\right) \mid\right) & \\
& \left.-\left(\pi_{A}^{+}\left(x_{j}\right)+\pi_{B}^{+}\left(x_{j}\right)\right)\right) \times 4^{-1}, \\
& +\left(\left|u_{A}^{+}\left(x_{j}\right)-u_{B}^{+}\left(x_{j}\right)\right|\right. \\
& \left.+\left|v_{A}^{+}\left(x_{j}\right)-v_{B}^{+}\left(x_{j}\right)\right|\right) \\
- & \left.\left(\pi_{A}^{-}\left(x_{j}\right)+\pi_{B}^{-}\left(x_{j}\right)\right)\right) \times 4^{-1} .
\end{aligned}
$$


And define

$$
\begin{aligned}
u_{M(A, B)}^{-}\left(x_{j}\right) & =\min \left\{M_{A B 1}\left(x_{j}\right), M_{A B 2}\left(x_{j}\right)\right\}, u_{M(A, B)}^{+}\left(x_{j}\right) \\
& =\max \left\{M_{A B 1}\left(x_{j}\right), M_{A B 2}\left(x_{j}\right)\right\}, \\
v_{M(A, B)}^{-}\left(x_{j}\right) & =\min \left\{M_{A B 3}\left(x_{j}\right), M_{A B 4}\left(x_{j}\right)\right\}, v_{M(A, B)}^{+}\left(x_{j}\right) \\
& =\max \left\{M_{A B 3}\left(x_{j}\right), M_{A B 4}\left(x_{j}\right)\right\} .
\end{aligned}
$$

Then, we get

$$
\begin{aligned}
M(A, B)=\{ & \left(x_{j},\left[u_{M(A, B)}^{-}\left(x_{j}\right), u_{M(A, B)}^{+}\left(x_{j}\right)\right],\right. \\
& {\left.\left.\left[v_{M(A, B)}^{-}\left(x_{j}\right), v_{M(A, B)}^{+}\left(x_{j}\right)\right]\right) \mid x_{j} \in X\right\} . }
\end{aligned}
$$

Theorem 5. Suppose that $E$ is an entropy measure for IVFS. The similarity measure on $\operatorname{IVFS}(X)$ is defined by $S(A, B)=$ $E(M(A, B))$ for each pair of IVFSs $A$ and $B$.

Proof. It is easy to prove that $E(M(A, B))$ satisfies the conditions listed in Definition 3.

(1) We can easily get $0 \leq E(M(A, B)) \leq 1$ since $0 \leq$ $E(A) \leq 1$ for all $A \in \operatorname{IVIFS}(X)$ and $M(A, B) \in$ $\operatorname{IVIFS}(X)$.

(2) With the definition of entropy and similarity measure, we can obtain

$E(M(A, B))=1$

$$
\begin{aligned}
& \Longleftrightarrow u_{M(A, B)}^{+}\left(x_{j}\right)=v_{M(A, B)}^{+}\left(x_{j}\right), \\
& u_{M(A, B)}^{-}\left(x_{j}\right)=v_{M(A, B)}^{-}\left(x_{j}\right), \\
& \forall x_{j} \in X \\
& \Longleftrightarrow u_{A}^{+}\left(x_{j}\right)=u_{B}^{+}\left(x_{j}\right) \text {, } \\
& v_{A}^{+}\left(x_{j}\right)=v_{B}^{+}\left(x_{j}\right) \text {, } \\
& u_{A}^{-}\left(x_{j}\right)=u_{B}^{-}\left(x_{j}\right) \text {, } \\
& v_{A}^{-}\left(x_{j}\right)=v_{B}^{-}\left(x_{j}\right), \quad \forall x_{j} \in X \\
& \Longleftrightarrow A=B
\end{aligned}
$$

(3) From the definition of $M(A, B)$, we can easily have that $M(A, B)=M(B, A)$. So it is obvious that $E(M(A, B))=E(M(B, A))$.
(4) As $A \subseteq B \subseteq C$, that is, $x_{j} \in X, v_{A}^{-}\left(x_{j}\right) \geq v_{B}^{-}\left(x_{j}\right) \geq$ $v_{C}^{-}\left(x_{j}\right), v_{A}^{+}\left(x_{j}\right) \geq v_{B}^{+}\left(x_{j}\right) \geq v_{C}^{+}\left(x_{j}\right), u_{A}^{-}\left(x_{j}\right) \leq u_{B}^{-}\left(x_{j}\right) \leq$ $u_{C}^{-}\left(x_{j}\right), u_{A}^{+}\left(x_{j}\right) \leq u_{B}^{+}\left(x_{j}\right) \leq u_{C}^{+}\left(x_{j}\right)$. We have

$$
\begin{aligned}
& M_{A C 1}\left(x_{j}\right) \\
&=\frac{1}{4}\left\{2+\left|u_{A}^{-}\left(x_{j}\right)-u_{C}^{-}\left(x_{j}\right)\right|+\left|v_{A}^{-}\left(x_{j}\right)-v_{C}^{-}\left(x_{j}\right)\right|\right.\left.\quad\left(\pi_{A}^{+}\left(x_{j}\right)+\pi_{C}^{+}\left(x_{j}\right)\right)\right\} \\
&=\frac{1}{2}\left(u_{C}^{-}\left(x_{j}\right)+v_{A}^{-}\left(x_{j}\right)\right), \\
& M_{A B 1}\left(x_{j}\right) \\
&=\frac{1}{4}\left\{2+\left|u_{A}^{-}\left(x_{j}\right)-u_{B}^{-}\left(x_{j}\right)\right|+\left|v_{A}^{-}\left(x_{j}\right)-v_{B}^{-}\left(x_{j}\right)\right|\right. \\
&\left.\quad-\left(\pi_{A}^{+}\left(x_{j}\right)+\pi_{B}^{+}\left(x_{j}\right)\right)\right\} \\
&=\frac{1}{2}\left(u_{B}^{-}\left(x_{j}\right)+v_{A}^{-}\left(x_{j}\right)\right) .
\end{aligned}
$$

So we can get $M_{A C 1}\left(x_{j}\right) \geq M_{A B 1}\left(x_{j}\right)$ because of $u_{B}^{-}\left(x_{j}\right) \leq$ $u_{C}^{-}\left(x_{j}\right)$.

Thus in the same way, we can easily prove that

$$
\begin{array}{ll}
M_{A C 1}\left(x_{j}\right) \geq M_{A B 1}\left(x_{j}\right), & M_{A C 2}\left(x_{j}\right) \geq M_{A B 2}\left(x_{j}\right), \\
M_{A C 3}\left(x_{j}\right) \leq M_{A B 3}\left(x_{j}\right), & M_{A C 4}\left(x_{j}\right) \leq M_{A B 4}\left(x_{j}\right) .
\end{array}
$$

We can also obtain

$$
\begin{aligned}
& u_{M(A, C)}^{-}\left(x_{j}\right) \geq u_{M(A, B)}^{-}\left(x_{j}\right), \\
& u_{M(A, C)}^{+}\left(x_{j}\right) \geq u_{M(A, B)}^{+}\left(x_{j}\right), \\
& v_{M(A, C)}^{-}\left(x_{j}\right) \leq v_{M(A, B)}^{-}\left(x_{j}\right), \\
& u_{M(A, C)}^{+}\left(x_{j}\right) \leq u_{M(A, B)}^{+}\left(x_{j}\right) .
\end{aligned}
$$

Therefore $M(A, B) \subseteq M(A, C)$.

Besides, we have $u_{M(A, B)}^{-}\left(x_{j}\right) \geq v_{M(A, B)}^{-}\left(x_{j}\right), u_{M(A, B)}^{+}\left(x_{j}\right) \geq$ $v_{M(A, B)}^{+}\left(x_{j}\right)$ by the definition of $M(A, B)$. We can get the result that

$$
E(M(A, B)) \geq E(M(A, C)), \quad S(A, B) \geq S(A, C) .
$$

In the same way, we can also have $E(M(B, C)) \geq E(M(A, C))$. 
Remark 6. Let $A=\{\langle x,[0.1,0.2],[0.5,0.6]\rangle\}$ and $B=$ $\{\langle x,[0.2,0.3],[0.5,0.6]\rangle\}$ be two IVIFSs. With (18), we can have

$$
\begin{gathered}
M_{A B 1}(x)=0.25 *(2+0.1-0.7)=0.35, \\
M_{A B 2}(x)=0.25 *(2+0.1-0.3)=0.45, \\
M_{A B 3}(x)=0.25 *(2-0.1-0.7)=0.3, \\
M_{A B 2}(x)=0.25 *(2-0.1-0.3)=0.4 . \\
u_{M(A, B)}^{-}(x)=\min \left\{M_{A B 1}(x), M_{A B 2}(x)\right\}=0.35, \\
u_{M(A, B)}^{+}(x)=\max \left\{M_{A B 1}(x), M_{A B 2}(x)\right\}=0.45, \\
v_{M(A, B)}^{-}(x)=\min \left\{M_{A B 3}(x), M_{A B 4}(x)\right\}=0.3, \\
v_{M(A, B)}^{+}(x)=\max \left\{M_{A B 3}(x), M_{A B 4}(x)\right\}=0.4 .
\end{gathered}
$$

Hence we obtain that $M(A, B)=\{\langle x,[0.35,0.45],[0.3,0.4]\rangle\}$.

We can get $E(M(A, B))=12 / 13$, if we chose the entropy formula of IVIFSs defined in Definition 2.

Corollary 7. Let $E$ be the entropy measure defined in Definition 2; that is, $A \in \operatorname{IVIFS}(X)$,

$E(A)$

$$
\begin{aligned}
=\frac{1}{n} \sum_{j=1}^{n}( & \min \left\{u_{A}^{-}\left(x_{j}\right), v_{A}^{-}\left(x_{j}\right)\right\}+\min \left\{u_{A}^{+}\left(x_{j}\right), v_{A}^{+}\left(x_{j}\right)\right\} \\
+ & \left.\pi_{A}^{-}\left(x_{j}\right)+\pi_{A}^{+}\left(x_{j}\right)\right) \\
\times & \max \left\{u_{A}^{-}\left(x_{j}\right), v_{A}^{-}\left(x_{j}\right)\right\} \\
& +\max \left\{u_{A}^{+}\left(x_{j}\right), v_{A}^{+}\left(x_{j}\right)\right\} \\
& \left.+\pi_{A}^{-}\left(x_{j}\right)+\pi_{A}^{+}\left(x_{j}\right)\right)^{-1} .
\end{aligned}
$$

Then the similarity measure is defined by

$$
S(A, B)=\frac{1}{n} \sum_{j=1}^{n} \frac{4-\left(u_{j}^{-}+u_{j}^{+}+v_{j}^{-}+v_{j}^{+}\right)+\left(\pi_{j}^{-}+\pi_{j}^{+}\right)}{4+\left(u_{j}^{-}+u_{j}^{+}+v_{j}^{-}+v_{j}^{+}\right)+\left(\pi_{j}^{-}+\pi_{j}^{+}\right)},
$$

for $A, B \in \operatorname{IVIFS}(X)$, where

$$
\begin{gathered}
u_{j}^{-}=\left|u_{A}^{-}\left(x_{j}\right)-u_{B}^{-}\left(x_{j}\right)\right|, \quad u_{j}^{+}=\left|u_{A}^{+}\left(x_{j}\right)-u_{B}^{+}\left(x_{j}\right)\right|, \\
v_{j}^{-}=\left|v_{A}^{-}\left(x_{j}\right)-v_{B}^{-}\left(x_{j}\right)\right|, \\
v_{j}^{+}=\left|v_{A}^{+}\left(x_{j}\right)-v_{B}^{+}\left(x_{j}\right)\right|, \quad \pi_{j}^{-}=\pi_{A}^{-}\left(x_{j}\right)+\pi_{B}^{-}\left(x_{j}\right), \\
\pi_{j}^{+}=\pi_{A}^{+}\left(x_{j}\right)+\pi_{B}^{+}\left(x_{j}\right) .
\end{gathered}
$$

Let $w=\left\{w_{1}, w_{2}, \ldots, w_{n}\right\}$ be the weight vector of elements $x_{j}(j=1,2, \ldots, n)$, where $w_{j} \geq 0, \sum_{j=1}^{n} w_{j}=1$.
Then we get the weighted similarity measure as follows:

$S(A, B)=\sum_{j=1}^{n} w_{j} \frac{4-\left(u_{j}^{-}+u_{j}^{+}+v_{j}^{-}+v_{j}^{+}\right)+\left(\pi_{j}^{-}+\pi_{j}^{+}\right)}{4+\left(u_{j}^{-}+u_{j}^{+}+v_{j}^{-}+v_{j}^{+}\right)+\left(\pi_{j}^{-}+\pi_{j}^{+}\right)}$.

Proof. We have $u_{M(A, B)}^{-}(x) \geq v_{M(A, B)}^{-}(x), u_{M(A, B)}^{+}(x) \geq$ $v_{M(A, B)}^{+}(x)$, with the definition of $M(A, B)$. Then we can obtain

$$
E(M(A, B))
$$

$$
\begin{aligned}
& =\frac{1}{n} \sum_{j=1}^{n}\left(\min \left\{u_{M(A, B)}^{-}\left(x_{j}\right), v_{M(A, B)}^{-}\left(x_{j}\right)\right\}\right. \\
& +\min \left\{u_{M(A, B)}^{+}\left(x_{j}\right), v_{M(A, B)}^{+}\left(x_{j}\right)\right\} \\
& \left.+\pi_{M(A, B)}^{-}\left(x_{j}\right)+\pi_{M(A, B)}^{+}\left(x_{j}\right)\right) \\
& \times\left(\max \left\{u_{M(A, B)}^{-}\left(x_{j}\right), v_{M(A, B)}^{-}\left(x_{j}\right)\right\}\right. \\
& +\max \left\{u_{M(A, B)}^{+}\left(x_{j}\right), v_{M(A, B)}^{+}\left(x_{j}\right)\right\} \\
& \left.+\pi_{M(A, B)}^{-}\left(x_{j}\right)+\pi_{M(A, B)}^{+}\left(x_{j}\right)\right)^{-1} \\
& =\frac{1}{n} \sum_{j=1}^{n}\left(v_{M(A, B)}^{-}\left(x_{j}\right)+v_{M(A, B)}^{+}\left(x_{j}\right)\right. \\
& \left.+\pi_{M(A, B)}^{-}\left(x_{j}\right)+\pi_{M(A, B)}^{+}\left(x_{j}\right)\right) \\
& \times\left(u_{M(A, B)}^{-}\left(x_{j}\right)+u_{M(A, B)}^{+}\left(x_{j}\right)\right. \\
& \left.+\pi_{M(A, B)}^{-}\left(x_{j}\right)+\pi_{M(A, B)}^{+}\left(x_{j}\right)\right)^{-1} \\
& =\frac{1}{n} \sum_{j=1}^{n} \frac{2-u_{M(A, B)}^{-}\left(x_{j}\right)-u_{M(A, B)}^{+}\left(x_{j}\right)}{2-v_{M(A, B)}^{-}\left(x_{j}\right)-v_{M(A, B)}^{+}\left(x_{j}\right)} \\
& =\frac{1}{n} \sum_{j=1}^{n} \frac{2-M_{A B 1}\left(x_{j}\right)-M_{A B 2}\left(x_{j}\right)}{2-M_{A B 3}(x)-M_{A B 4}(x)} .
\end{aligned}
$$

If we let

$$
\begin{gathered}
u_{j}^{-}=\left|u_{A}^{-}\left(x_{j}\right)-u_{B}^{-}\left(x_{j}\right)\right|, \quad u_{j}^{+}=\left|u_{A}^{+}\left(x_{j}\right)-u_{B}^{+}\left(x_{j}\right)\right|, \\
v_{j}^{-}=\left|v_{A}^{-}\left(x_{j}\right)-v_{B}^{-}\left(x_{j}\right)\right|, \\
v_{j}^{+}=\left|v_{A}^{+}\left(x_{j}\right)-v_{B}^{+}\left(x_{j}\right)\right|, \quad \pi_{j}^{-}=\pi_{A}^{-}\left(x_{j}\right)+\pi_{B}^{-}\left(x_{j}\right), \\
\pi_{j}^{+}=\pi_{A}^{+}\left(x_{j}\right)+\pi_{B}^{+}\left(x_{j}\right),
\end{gathered}
$$

then

$$
E(M(A, B))=\frac{1}{n} \sum_{j=1}^{n} \frac{4-\left(u_{j}^{-}+u_{j}^{+}+v_{j}^{-}+v_{j}^{+}\right)+\left(\pi_{j}^{-}+\pi_{j}^{+}\right)}{4+\left(u_{j}^{-}+u_{j}^{+}+v_{j}^{-}+v_{j}^{+}\right)+\left(\pi_{j}^{-}+\pi_{j}^{+}\right)} .
$$


Now we let $w=\left\{w_{1}, w_{2}, \ldots, w_{n}\right\}$ be the weight vector of elements $x_{j}(j=1,2, \ldots, n)$; the weighted similarity measure can be written by the form

$$
E(M(A, B))=\sum_{j=1}^{n} w_{j} \frac{4-\left(u_{j}^{-}+u_{j}^{+}+v_{j}^{-}+v_{j}^{+}\right)+\left(\pi_{j}^{-}+\pi_{j}^{+}\right)}{4+\left(u_{j}^{-}+u_{j}^{+}+v_{j}^{-}+v_{j}^{+}\right)+\left(\pi_{j}^{-}+\pi_{j}^{+}\right)} .
$$

The function $S$ defined by (30) is a similarity measure on $\operatorname{IVIFS}(X)$, from Theorem 5.

\subsection{Comparison with Some Existing Similarity Measures}

Example 8. We test the new similarity measure by Example 4 given in Section 2.3. Consider $A=\{x, 0.5,0.5\}, B=$ $\{x, 0.3,0.7\}$, and $C=\{x, 0.3,0.3\}$. From (18), we can have

$$
\begin{aligned}
M_{A B 1}= & M_{A B 2} \\
= & 0.25 *\left\{2+\left|u_{A}(x)-u_{B}(x)\right|+\left|v_{A}(x)-v_{B}(x)\right|\right. \\
& \left.\quad-\left(\pi_{A}(x)+\pi_{B}(x)\right)\right\}=0.6, \\
M_{A B 3}= & M_{A B 4} \\
= & 0.25 *\left\{2-\left(\left|u_{A}(x)-u_{B}(x)\right|+\left|v_{A}(x)-v_{B}(x)\right|\right)\right. \\
& \left.\quad-\left(\pi_{A}(x)+\pi_{B}(x)\right)\right\}=0.4 .
\end{aligned}
$$

Then we can get $M(A, B)=\{x, 0.6,0.4\}$. The similarity of $A$ and $B$ is

$$
S(A, B)=E(M(A, B))=\frac{2}{3}
$$

by (30). In the same way, we can easily get

$$
M(A, C)=\{x, 0.5,0.3\}, \quad S(A, C)=E(M(A, C))=\frac{5}{7} .
$$

So we can get the right answer that $A$ is more similar to $C$ than to $B$; that is, $S(A, B)<S(A, C)$. While using the similarity measures $S_{1}, S_{2}, S_{3}, S_{4}$, and $S_{5}$, we can get the result $S(A, B)=S(A, C)$ from Section 2.3. It shows that the new similarity measure is much more reasonable than the $S_{1}, S_{2}$, $S_{3}, S_{4}, S_{5}$.

In [32], Hung and Yang proposed three similarity measures between IFSs. Let $A, B \in \operatorname{IFSs}(X)$ and $d(A, B)$ is the distance between two IFSs. Consider

$$
\begin{gathered}
S_{6}(A, B)=1-d(A, B), \\
S_{7}(A, B)=\frac{1-d(A, B)}{1+d(A, B)}, \\
S_{8}(A, B)=\frac{e^{-d(A, B)}-e^{-1}}{1-e^{-1}},
\end{gathered}
$$

where $d(A, B)=(1 / n) \sum_{j=1}^{n} \max \left\{\left|u_{A}\left(x_{j}\right)-u_{B}\left(x_{j}\right)\right|, \mid v_{A}\left(x_{j}\right)-\right.$ $\left.v_{B}\left(x_{j}\right) \mid\right\}$.
The following example shows some drawbacks of formulas (38).

Example 9. Let $A=\{x, 0.5,0.5\}, B=\{x, 0.4,0.6\}$, and $C=$ $\{x, 0.4,0.5\}$ be three IFSs.

Intuitively, one can see that $A$ is more similar to $C$ than to $B$. However, using the similarity measures given by (38), we have

$$
\begin{aligned}
& d(A, B)=\max \{|0.5-0.4|,|0.5-0.6|\}=0.1, \\
& d(A, C)=\max \{|0.5-0.4|,|0.5-0.5|\}=0.1 .
\end{aligned}
$$

So $S_{6}(A, B)=S_{6}(A, C), S_{7}(A, B)=S_{7}(A, C), S_{8}(A, B)=$ $S_{8}(A, C)$, because of $d(A, B)=d(A, C)$. Now we can calculate the similarity measures $S(A, B)$ and $S(A, C)$ by formula (30). We can get

$$
\begin{gathered}
M_{A B 1}=M_{A B 2}=0.25 *(2+0.1+0.1-0)=0.55, \\
M_{A B 3}=M_{A B 4}=0.25 *(2-0.1-0.1-0)=0.45, \\
M(A, B)=\{x, 0.55,0.45\}, \\
S(A, B)=E(M(A, B))=\frac{9}{11}, \\
M_{A C 1}=M_{A C 2}=0.25 *(2+0+0.1-0.1)=0.5, \\
M_{A C 3}=M_{A C 4}=0.25 *(2-0-0.1-0.1)=0.45, \\
M(A, C)=\{x, 0.5,0.45\}, \\
S(A, C)=E(M(A, C))=\frac{10}{11}, \\
S(A, C)>S(A, B),
\end{gathered}
$$

which means that $A$ is more similar to $C$ than to $B$, consistent with the intuition. Therefore, the new similarity measure (30) is proved to be more reasonable than $S_{6}, S_{7}, S_{8}$ in some cases.

Let $X=\left\{x_{1}, x_{2}, \ldots, x_{n}\right\}$ and $A, B \in \operatorname{IFSs}(X)$. Chen [33] presented the following similarity measures between IFSs $A$ and $B$ :

$$
\begin{aligned}
& S_{9}(A, B) \\
& =1-\frac{\sum_{j=1}^{n}\left|u_{A}\left(x_{j}\right)-v_{A}\left(x_{j}\right)-\left(u_{B}\left(x_{j}\right)-v_{B}\left(x_{j}\right)\right)\right|}{2 n}, \\
& \quad S_{10}(A, B)=1-\sqrt{\frac{\sum_{j=1}^{n}\left|\psi_{A}\left(x_{j}\right)-\psi_{B}\left(x_{j}\right)\right|^{q}}{n}},
\end{aligned}
$$

where $1 \leq q \leq \infty$, and, for each $j$,

$$
\begin{aligned}
& \psi_{A}\left(x_{j}\right)=0.5\left(u_{A}\left(x_{j}\right)-v_{A}\left(x_{j}\right)+1\right), \\
& \psi_{B}\left(x_{j}\right)=0.5\left(u_{B}\left(x_{j}\right)-v_{B}\left(x_{j}\right)+1\right) .
\end{aligned}
$$

The following example indicates that the above similarity measures $S_{9}, S_{10}$ are also not reasonable in some cases. 
Example 10. Let $A=\{x, 0.5,0.5\}, B=\{x, 0.2,0.2\}$, and $C=\{x, 0.49,0.51\}$ be three IFSs. It is obvious that $A$ is more similar to $C$ than to $B$.

We calculate the degree of similarity between $A$ and $B$, as well as $A$ and $C$ using the new similarity measure given by (30). Then $S(A, B)=5 / 6, S(A, C)=99 / 101$, which show that $A$ is more similar to $C$ than to $B$ and is consistent with intuition. But if we use formula (41) to calculate the similarity measure, then

$$
\begin{gathered}
S_{9}(A, B)=1-0.5 *|0.5-0.5-(0.2-0.2)|=1, \\
S_{9}(A, C)=1-0.5 *|0.5-0.5-(0.49-0.51)|=0.99, \\
\psi_{A}\left(x_{j}\right)=0.5, \quad \psi_{B}\left(x_{j}\right)=0.5, \quad \psi_{C}\left(x_{j}\right)=0.49, \\
S_{10}(A, B)=1-\sqrt{\frac{|0.5-0.5|^{q}}{1}}=1, \\
S_{10}(A, C)=1-\sqrt{\frac{|0.5-0.49|^{q}}{1}}=0.99, \\
S_{9}(A, B)>S_{9}(A, C), \quad S_{10}(A, B)>S_{10}(A, C),
\end{gathered}
$$

which is not reasonable. So the new similarity measure is illustrated to be more effective than formula (41).

Let $X=\left\{x_{1}, x_{2}, \ldots, x_{n}\right\}$ and $A, B \in \operatorname{IFSs}(X)$. Xu [28] gave many similarity measures based on the distance measure. The weight of each element is $w_{j}, j=1,2, \ldots, n$. Consider

$$
\begin{aligned}
& S_{11}(A, B) \\
& =1-\left[\frac { 1 } { 2 } \sum _ { j = 1 } ^ { n } w _ { j } \left(\left|u_{A}\left(x_{j}\right)-u_{B}\left(x_{j}\right)\right|^{\alpha}\right.\right. \\
& +\left|v_{A}\left(x_{j}\right)-v_{B}\left(x_{j}\right)\right|^{\alpha} \\
& \left.\left.+\left|\pi_{A}\left(x_{j}\right)-\pi_{B}\left(x_{j}\right)\right|^{\alpha}\right)\right]^{1 / \alpha},
\end{aligned}
$$

$S_{12}(A, B)$

$$
\begin{aligned}
=1-\left[\left(\sum_{j=1}^{n} w_{j}(\right.\right. & \left|u_{A}\left(x_{j}\right)-u_{B}\left(x_{j}\right)\right|^{\alpha} \\
& +\left|v_{A}\left(x_{j}\right)-v_{B}\left(x_{j}\right)\right|^{\alpha} \\
& \left.\left.+\left|\pi_{A}\left(x_{j}\right)-\pi_{B}\left(x_{j}\right)\right|^{\alpha}\right)\right)
\end{aligned}
$$

$$
\begin{aligned}
\times\left(\sum _ { j = 1 } ^ { n } w _ { j } \left(\left|u_{A}\left(x_{j}\right)+u_{B}\left(x_{j}\right)\right|^{\alpha}\right.\right. \\
+\left|v_{A}\left(x_{j}\right)+v_{B}\left(x_{j}\right)\right|^{\alpha} \\
\left.\left.\left.+\left|\pi_{A}\left(x_{j}\right)+\pi_{B}\left(x_{j}\right)\right|^{\alpha}\right)\right)^{-1}\right]^{1 / \alpha},
\end{aligned}
$$

$S_{13}(A, B)$

$$
\begin{aligned}
=\left(\sum_{j=1}^{n} w_{j}(\right. & \min \left(u_{A}\left(x_{j}\right), u_{B}\left(x_{j}\right)\right) \\
& +\min \left(v_{A}\left(x_{j}\right), v_{B}\left(x_{j}\right)\right) \\
& \left.\left.+\min \left(\pi_{A}\left(x_{j}\right), \pi_{B}\left(x_{j}\right)\right)\right)\right) \\
\times\left(\sum _ { j = 1 } ^ { n } w _ { j } \left(\max \left(u_{A}\left(x_{j}\right), u_{B}\left(x_{j}\right)\right)\right.\right. & \left.+\max \left(v_{A}\left(x_{j}\right), v_{B}\left(x_{j}\right)\right)\right)
\end{aligned}
$$$$
\left.\left.+\max \left(\pi_{A}\left(x_{j}\right), \pi_{B}\left(x_{j}\right)\right)\right)\right)^{-1},
$$

$$
\begin{aligned}
& S_{14}(A, B) \\
& =\left(\sum _ { j = 1 } ^ { n } w _ { j } \left(u_{A}\left(x_{j}\right) * u_{B}\left(x_{j}\right)+v_{A}\left(x_{j}\right) * v_{B}\left(x_{j}\right)\right.\right. \\
& \left.\left.+\pi_{A}\left(x_{j}\right) * \pi_{B}\left(x_{j}\right)\right)\right) \\
& \times\left(\operatorname { m a x } \left(\sum_{j=1}^{n} w_{j}\left(u_{A}^{2}\left(x_{j}\right)+v_{A}^{2}\left(x_{j}\right)+\pi_{A}^{2}\left(x_{j}\right)\right),\right.\right. \\
& \left.\left.\sum_{j=1}^{n} w_{j}\left(u_{B}^{2}\left(x_{j}\right)+v_{B}^{2}\left(x_{j}\right)+\pi_{B}^{2}\left(x_{j}\right)\right)\right)\right)^{-1} .
\end{aligned}
$$

Example 11. Let $A=\{x, 0.5,0.5\}, B=\{x, 0.3,0.7\}$, and $C=$ $\{x, 0.3,0.4\}$ be three IFSs.

For IFSs $A, B$, and $C$, we have the following results using the similarity measures $S_{11}, S_{12}, S_{13}, S_{14}$ :

$$
\begin{array}{cc}
S_{11}(A, B)=0.8, & S_{11}(A, C)=0.735, \\
S_{12}(A, B)=0.804, & S_{12}(A, C)=0.698, \\
S_{12}(A, B)=0.804, & S_{12}(A, C)=0.698, \\
S_{14}(A, B)=0.862, & S_{14}(A, C)=0.7 .
\end{array}
$$


It indicates that $A$ is more similar to $B$ than to $C$. As the membership of $B$ and $C$ is the same, we only analyse the change of the membership. Comparing $A$ with $B$, the change degree of nonmembership is from 0.5 to 0.7 . While comparing $C$ with $A$, the change degree of nonmembership is from 0.4 to 0.5 . Hence, intuitively, $A$ is more similar to $C$ than to $B$ and the results of $S_{11}, S_{12}, S_{13}, S_{14}$ are not reasonable. Now we calculate the similarity measures $S(A, B)$ and $S(A, C)$ by formula (30). Then $S(A, B)=2 / 3$ and $S(A, C)=19 / 27$, which indicated that $A$ is more similar to $C$ than to $B$ and is consistent with intuition.

Later, Xia and $\mathrm{Xu}$ [29] also proposed the following similarity measures. Xu and Yager [27] named $\alpha_{r}=\left(u_{\alpha_{r}}, v_{\alpha_{r}}\right)$ an intuitionistic fuzzy value (IFV). Let $\alpha=\left(\alpha_{1}, \alpha_{2}, \ldots, \alpha_{n}\right)$ and $\beta=\left(\beta_{1}, \beta_{2}, \ldots, \beta_{n}\right)$ be two collections of IFVs and $\kappa, \lambda \in$ $[0,1], t_{a_{j}}=u_{\alpha_{j}}-u_{\beta_{j}}, t_{b_{j}}=v_{\alpha_{j}}-v_{\beta_{j}}, t_{c_{j}}=\pi_{\alpha_{j}}-\pi_{\beta_{j}}=-t_{a_{j}}-$ $t_{b_{j}}, j=(1,2, \ldots, n)$. Then the author defined the similarity measures as

$$
S_{D_{\kappa}}^{\eta}(\alpha, \beta)=1-\left[\sum_{j=1}^{n} w_{j}\left(\left|(1-\kappa) t_{a_{j}}-\kappa t_{b_{j}}\right|^{\eta}\right)\right]^{1 / \eta} .
$$

For $\kappa+\lambda \leq 1$,

$$
\begin{gathered}
S_{F_{\kappa, \lambda}}^{\eta}(\alpha, \beta) \\
=1-\left[\frac { 1 } { 2 } \sum _ { j = 1 } ^ { n } w _ { j } \left(\left|(1-\kappa) t_{a_{j}}-\kappa t_{b_{j}}\right|^{\eta}+\left|(1-\lambda) t_{a_{j}}-\lambda t_{b_{j}}\right|^{\eta}\right.\right. \\
\left.\left.+\left|(1-\kappa-\lambda)\left(t_{a_{j}}+t_{b_{j}}\right)\right|^{\eta}\right)\right]^{1 / \eta}
\end{gathered}
$$

$S_{G_{\kappa, \lambda}}^{\eta}(\alpha, \beta)$

$$
=1-\left[\frac{1}{2} \sum_{j=1}^{n} w_{j}\left(\left|\kappa t_{a_{j}}\right|^{\eta}+\left|\lambda t_{b_{j}}\right|^{\eta}+\left|\kappa t_{a_{j}}+\lambda t_{b_{j}}\right|^{\eta}\right)\right]^{1 / \eta},
$$

$$
\begin{aligned}
& S_{H_{\kappa, \lambda}}^{\eta}(\alpha, \beta) \\
& =1-\left[\frac { 1 } { 2 } \sum _ { j = 1 } ^ { n } w _ { j } \left(\left|\kappa t_{a_{j}}\right|^{\eta}+\left|\lambda t_{a_{j}}-(1-\lambda) t_{b_{j}}\right|^{\eta}\right.\right. \\
& \left.\left.+\left|(\kappa-\lambda) t_{a_{j}}+(1-\lambda) t_{b_{j}}\right|^{\eta}\right)\right]^{1 / \eta},
\end{aligned}
$$

$S_{H_{\kappa, \lambda}^{*}}^{\eta}(\alpha, \beta)$

$$
\begin{array}{r}
=1-\left[\frac { 1 } { 2 } \sum _ { j = 1 } ^ { n } w _ { j } \left(\left|\kappa t_{a_{j}}\right|^{\eta}+\left|\kappa \lambda t_{a_{j}}-(1-\lambda) t_{b_{j}}\right|^{\eta}\right.\right. \\
\left.\left.+\left|(1-\lambda)\left(\kappa t_{a_{j}}+t_{b_{j}}\right)\right|^{\eta}\right)\right]^{1 / \eta},
\end{array}
$$

$$
\begin{aligned}
& S_{J_{\kappa, \lambda}}^{\eta}(\alpha, \beta) \\
& =1-\left[\frac { 1 } { 2 } \sum _ { j = 1 } ^ { n } w _ { j } \left(\left|(1-\kappa) t_{a_{j}}-\kappa t_{b_{j}}\right|^{\eta}+\left|\lambda t_{b_{j}}\right|\right.\right. \\
& \left.\left.+\left|(1-\kappa) t_{a_{j}}+(\lambda-\kappa) t_{b_{j}}\right|^{\eta}\right)\right]^{1 / \eta},
\end{aligned}
$$

$S_{J_{\kappa, \lambda}^{*}}^{\eta}(\alpha, \beta)$

$$
\begin{array}{r}
=1-\left[\frac { 1 } { 2 } \sum _ { j = 1 } ^ { n } w _ { j } \left(\left|(1-\kappa) t_{a_{j}}-\kappa \lambda t_{b_{j}}\right|^{\eta}+\left|\lambda t_{b_{j}}\right|\right.\right. \\
\left.\left.+\left|(1-\kappa)\left(t_{a_{j}}+\lambda t_{b_{j}}\right)\right|^{\eta}\right)\right]^{1 / \eta} .
\end{array}
$$

For $\kappa+\lambda \leq 1$,

$$
\begin{aligned}
& S_{P_{\kappa, \lambda}}^{\eta}(\alpha, \beta) \\
& =1-\left[\frac { 1 } { 2 } \sum _ { j = 1 } ^ { n } w _ { j } \left(\left|\max \left(\kappa, u_{\alpha_{j}}\right)-\max \left(\kappa, u_{\beta_{j}}\right)\right|^{\eta}\right.\right. \\
& +\left|\min \left(\lambda, v_{\alpha_{j}}\right)-\min \left(\lambda, v_{\beta_{j}}\right)\right|^{\eta} \\
& +\mid \max \left(\kappa, u_{\alpha_{j}}\right)-\max \left(\kappa, u_{\beta_{j}}\right) \\
& \left.\left.+\min \left(\lambda, v_{\alpha_{j}}\right)-\left.\min \left(\lambda, v_{\beta_{j}}\right)\right|^{\eta}\right)\right]^{1 / \eta} .
\end{aligned}
$$

For $\kappa+\lambda \leq 1$,

$$
\begin{aligned}
& S_{\mathrm{Q}_{k, \lambda}}^{\eta}(\alpha, \beta) \\
& =1-\left[\frac { 1 } { 2 } \sum _ { j = 1 } ^ { n } w _ { j } \left(\left|\min \left(\kappa, u_{\alpha_{j}}\right)-\min \left(\kappa, u_{\beta_{j}}\right)\right|^{\eta}\right.\right. \\
& +\left|\max \left(\lambda, v_{\alpha_{j}}\right)-\max \left(\lambda, v_{\beta_{j}}\right)\right|^{\eta} \\
& +\mid \min \left(\kappa, u_{\alpha_{j}}\right)-\min \left(\kappa, u_{\beta_{j}}\right) \\
& \left.\left.+\max \left(\lambda, v_{\alpha_{j}}\right)-\left.\max \left(\lambda, v_{\beta_{j}}\right)\right|^{\eta}\right)\right]^{1 / \eta} .
\end{aligned}
$$

Example 12. Let $A=\{x, 0.3,0.3\}, B=\{x, 0.4,0.4\}$, and $C=$ $\{x, 0.4,0.5\}$ be three IFVs. It is clear to see that $A$ is much more similar to $B$ than to $C$. However, using the similarity measure 
(46), we can get $S_{D_{0.2}}^{5}(A, B)=0.94, S_{D_{0.2}}^{5}(A, C)=0.96$. Hence, $S_{D_{0.2}}^{5}(A, B)<S_{D_{0.2}}^{5}(A, C)$, which is not reasonable. If we use the new similarity measure (30), we have $S(A, B)=0.8571$, $S(A, C)=0.7857$. Therefore, the new similarity measure is illustrated to be more reasonable than $S_{D_{\kappa}}^{\eta}$.

Example 13. Let $A=\{x, 0.5,0.5\}, B=\{x, 0.2,0.4\}$, and $C=$ $\{x, 0.2,0.65\}$ be three IFVs. We can get the following results using formula (47). We adopt the values of $\eta, \kappa, \lambda$ from [31]

$$
\begin{gathered}
S_{F_{0.2,0.1}}^{5}(A, B)=0.7203, \quad S_{F_{0.2,0.1}}^{5}(A, C)=0.7219, \\
S_{G_{0.7,0.8}}^{5}(A, B)=0.7381, \quad S_{G_{0.7,0.8}}^{5}(A, C)=0.8145, \\
S_{H_{0.8,0.3}}^{5}(A, B)=0.7691, \quad S_{H_{0.8,0.3}}^{5}(A, C)=0.778, \\
S_{H_{0.9,0.2}^{*}}^{5}(A, B)=0.7158, \quad S_{H_{0.9,0.2}^{*}}^{5}(A, C)=0.7597, \\
S_{J_{0.1,0.6}}^{5}(A, B)=0.704, \quad S_{J_{0.1,0.6}}^{5}(A, C)=0.7447, \\
S_{J_{0.2,0.7}^{*}}^{5}(A, B)=0.7301, \quad S_{J_{0.2,0.7}^{*}}^{5}(A, C)=0.769 .
\end{gathered}
$$

For similarity measures (48)-(49), the results are varying when the values of $\kappa, \lambda$ are different. For example, let $\kappa=0$; we can get the following:

$$
\begin{array}{cc}
S_{P_{\kappa, \lambda}}^{\eta}(A, B) \leq S_{P_{\kappa, \lambda}}^{\eta}(A, C), & \text { if } \lambda \leq 0.6, \\
S_{P_{\kappa, \lambda}}^{\eta}(A, B)>S_{P_{\kappa, \lambda}}^{\eta}(A, C), & \text { if } \lambda>0.6, \\
S_{Q_{\kappa, \lambda}}^{\eta}(A, B) \geq S_{Q_{\kappa, \lambda}}^{\eta}(A, C), & \text { if } \lambda \leq 0.65, \\
S_{Q_{\kappa, \lambda}}^{\eta}(A, B)=S_{Q_{\kappa, \lambda}}^{\eta}(A, C), & \text { if } \lambda>0.65 .
\end{array}
$$

If we use formula (30) proposed in this paper, we can get $S(A, B)=0.7143$ and $S(A, C)=0.6642$. Furthermore, the change of the membership from $A$ to $B$ and $A$ to $C$ is the same. But the change degree of nonmembership is different. Comparing $A$ with $B$, the change is from 0.5 to 0.4 which is smaller than that comparing $A$ with $C$. Accordingly, $A$ is much more similar to $B$ than to $C$ and our similarity measure is more effective in some cases.

\section{Application to Expert System}

IVIFS is a very suitable tool to the expert system to process the imperfect information. We apply the new similarity measure defined by (30) to some applications.

\subsection{Pattern Recognition}

Step 1. Suppose that there exist $m$ patterns which are represented by IVIFSs for a pattern recognition problem

$$
\begin{aligned}
A_{i}=\{ & \left(x_{j},\left[u_{A_{i}}^{-}\left(x_{j}\right), u_{A_{i}}^{+}\left(x_{j}\right)\right],\right. \\
& {\left.\left.\left[v_{A i}^{-}\left(x_{j}\right), v_{A i}^{+}\left(x_{j}\right)\right]\right) \mid x_{j} \in X\right\}, }
\end{aligned}
$$

for $i=1,2, \ldots, m$, in the feature space $X=\left\{x_{1}, x_{2}, \ldots, x_{n}\right\}$, and suppose that there is a sample to be recognized which is represented by IVIFSs

$$
B=\left\{\left(x_{j},\left[u_{B}^{-}\left(x_{j}\right), u_{B}^{+}\left(x_{j}\right)\right],\left[v_{B}^{-}\left(x_{j}\right), v_{B}^{+}\left(x_{j}\right)\right]\right) \mid x_{j} \in X\right\} .
$$

Step 2. Calculate the similarity degree $S\left(A_{i}, B\right)$ between $A_{i}$ and $B$ by formula (30).

Step 3. Select the largest $S\left(A_{k}, B\right)$, from $S\left(A_{i}, B\right), i=$ $1,2, \ldots, m$. According to the principle of maximum similarity degree between IVIFSs, the sample $B$ belongs to the pattern $A_{k}$.

Example 14 (see [32]). Assume that there are three patterns denoted by IFSs in $X=\left\{x_{1}, x_{2}, x_{3}\right\}$

$$
\begin{aligned}
& A_{1}=\left\{\left(x_{1}, 0.1,0.1\right),\left(x_{2}, 0.5,0.1\right),\left(x_{3}, 0.1,0.9\right)\right\}, \\
& A_{2}=\left\{\left(x_{1}, 0.5,0.5\right),\left(x_{2}, 0.7,0.3\right),\left(x_{3}, 0.0,0.8\right)\right\}, \\
& A_{3}=\left\{\left(x_{1}, 0.7,0.2\right),\left(x_{2}, 0.1,0.8\right),\left(x_{3}, 0.4,0.4\right)\right\} .
\end{aligned}
$$

Assume that a sample $B=\left\{\left(x_{1}, 0.4,0.4\right),\left(x_{2}, 0.6,0.2\right)\right.$, $\left.\left(x_{3}, 0.0,0.8\right)\right\}$ is given and let the weight vector $w=$ $(1 / 3,1 / 3,1 / 3)^{T}$ and $q=2$. According to formula (30) and the similarity measures $S_{9}, S_{10}$ proposed by Chen [33], the following results can be obtained:

$$
\begin{gathered}
S\left(A_{1}, B\right)=0.7857, \quad S\left(A_{2}, B\right)=0.8889, \\
S\left(A_{3}, B\right)=0.5097, \\
S_{9}\left(A_{1}, B\right)=1, \quad S_{9}\left(A_{2}, B\right)=1, \\
S_{9}\left(A_{3}, B\right)=0.6, \\
S_{10}\left(A_{1}, B\right)=1, \quad S_{10}\left(A_{2}, B\right)=1, \\
S_{10}\left(A_{3}, B\right)=0.5817 .
\end{gathered}
$$

We can see that the similarity measures $S_{9}, S_{10}$ cannot recognize which pattern $B$ belongs to. Based on the principle of maximum degree of similarity, it is obvious that the sample $B$ belongs to $A_{2}$ using our similarity measure and the results are the same with some other similarity measures [32].

Example 15 (see [34]). We discuss the medical diagnosis problem. Let us consider a set of diagnoses $A=$ $\left\{A_{1}\right.$ (Viralfever), $A_{2}$ (Malaria), $A_{3}$ (Typhoid) $\}$ as well as a set of symptoms $X=\left\{x_{1}\right.$ (Temprature), $x_{2}$ (Headache), $x_{3}$ (Cough) $\}$. Assume that a patient can be represented as follows:

$$
\begin{aligned}
B=\left\{\left(x_{1},[0.6,0.8],[0.1,0.2]\right),\left(x_{2},[0.3,0.7],[0.2,0.3]\right),\right. \\
\\
\left.\left(x_{3},[0.6,0.8],[0.1,0.2]\right)\right\} .
\end{aligned}
$$


Let the weight vector $w=(1 / 3,1 / 3,1 / 3)^{T}$ and each diagnosis can be denoted by IVIFSs as follows:

$$
\begin{aligned}
& A_{1}=\left\{\left(x_{1},[0.4,0.5],[0.3,0.4]\right),\left(x_{2},[0.4,0.6],[0.2,0.4]\right),\right. \\
&\left.\left(x_{3},[0.4,0.8],[0.1,0.2]\right)\right\}, \\
& A_{2}=\left\{\left(x_{1},[0.3,0.6],[0.3,0.4]\right),\left(x_{2},[0.5,0.6],[0.3,0.4]\right),\right. \\
&\left.\quad\left(x_{3},[0.4,0.5],[0.1,0.3]\right)\right\}, \\
& A_{3}=\left\{\left(x_{1},[0.7,0.8],[0.1,0.2]\right),\left(x_{2},[0.6,0.7],[0.1,0.3]\right),\right. \\
&\left.\left(x_{3},[0.3,0.4],[0.1,0.2]\right)\right\} .
\end{aligned}
$$

Our goal is to categorize the patient $B$ in one of the classes $A_{1}, A_{2}, A_{3}$. Then, we can have the following results based on formula (30) and (10)-(13):

$$
\begin{aligned}
& S\left(A_{1}, B\right)=0.8366, \quad S\left(A_{2}, B\right)=0.7586, \\
& S\left(A_{3}, B\right)=0.8577 \text {. } \\
& S_{2}\left(A_{1}, B\right)=0.8834, \quad S\left(A_{2}, B\right)=0.8334 \text {, } \\
& S\left(A_{3}, B\right)=0.9 \text {. } \\
& S_{3}\left(A_{1}, B\right)=0.517, \quad S_{3}\left(A_{2}, B\right)=0.4084 \text {, } \\
& S_{3}\left(A_{3}, B\right)=0.8292 \text {. } \\
& S_{4}\left(A_{1}, B\right)=0.8, \quad S_{4}\left(A_{2}, B\right)=0.7334, \\
& S_{4}\left(A_{3}, B\right)=0.7334 \text {. } \\
& S_{5}\left(A_{1}, B\right)=0.784, \quad S_{5}\left(A_{2}, B\right)=0.7292 \text {, } \\
& S_{5}\left(A_{3}, B\right)=0.7056 \text {. }
\end{aligned}
$$

Based on the recognition principle of the maximum similarity degree between the IVIFSs, we can get result that the similarity degree between $A_{3}$ and $B$ is the largest using formula (30), (10), and (11). Consider

$$
\begin{gathered}
S\left(A_{3}, B\right)=\max \left(S\left(A_{1}, B\right), S\left(A_{2}, B\right), S\left(A_{3}, B\right)\right), \\
S_{2}\left(A_{3}, B\right)=\max \left(S_{2}\left(A_{1}, B\right), S_{2}\left(A_{2}, B\right), S_{2}\left(A_{3}, B\right)\right), \\
S_{3}\left(A_{3}, B\right)=\max \left(S_{3}\left(A_{1}, B\right), S_{3}\left(A_{2}, B\right), S_{3}\left(A_{3}, B\right)\right) .
\end{gathered}
$$

It is the right answer that $B$ belongs to $A_{3}$, which is the same as in [34]. But the similarity measures $S_{4}, S_{5}$ would have the wrong answer. Thus, we can assign the patient to diagnosis $A_{3}$ according to the recognition of principle. So we can diagnose that the illness of the patient is typhoid.

The application of pattern recognition in medical diagnosis problem can be very significant. We can take advantage of the similarity measure to help us to diagnose the patient based on the database of the symptoms of illnesses. So it can help the patients much faster to know their illness, as well as alleviating the burden of doctors.
Example 16 (see [24]). Assume that there are four classes of building materials $A=$ $\left\{A_{1}\right.$ (poor), $A_{2}$ (average), $A_{3}$ (good), $A_{4}$ (excellent) $\}$ that are written in the form of IVIFSs $A_{i}=$ $\left\{\left(x_{j},\left[u_{A_{i}}^{-}\left(x_{j}\right), u_{A_{i}}^{+}\left(x_{j}\right)\right],\left[v_{A_{i}}^{-}\left(x_{j}\right), v_{A_{i}}^{+}\left(x_{j}\right)\right]\right) \mid x_{j} \in X\right\}(i=$ $1,2,3,4)$ in the future space $X=\left\{x_{1}, x_{2}, \ldots, x_{12}\right\}$ that denote twelve different indicators. And the weight vector $w$ is as follows:

$$
\begin{gathered}
w=(0.1,0.05,0.08,0.06,0.03,0.07,0.09, \\
0.12,0.15,0.07,0.13,0.05)^{T} .
\end{gathered}
$$

And there are an unknown building material $B$ and four classes of building materials represented by IVIFSs with respect to all indicators as follows:

$$
\begin{aligned}
& A_{1}=\left\{\left(x_{1},[0.1,0.2],[0.5,0.6]\right),\left(x_{2},[0.1,0.2],[0.7,0.8]\right),\right. \\
&\left(x_{3},[0.5,0.6],[0.3,0.4]\right),\left(x_{4},[0.8,0.9],[0.0,0.1]\right), \\
&\left(x_{5},[0.4,0.5],[0.3,0.4]\right),\left(x_{6},[0.0,0.1],[0.8,0.9]\right), \\
&\left(x_{7},[0.3,0.4],[0.5,0.6]\right),\left(x_{8},[1.0,1.0],[0.0,0.0]\right), \\
&\left(x_{9},[0.2,0.3],[0.6,0.7]\right),\left(x_{10},[0.4,0.5],[0.4,0.5]\right), \\
&\left.\left(x_{11},[0.7,0.8],[0.1,0.2]\right),\left(x_{12},[0.4,0.5],[0.4,0.5]\right)\right\}, \\
& A_{2}=\left\{\left(x_{1},[0.5,0.6],[0.3,0.4]\right),\left(x_{2},[0.6,0.7],[0.1,0.2]\right),\right. \\
&\left(x_{3},[1.0,1.0],[0.0,0.0]\right),\left(x_{4},[0.1,0.2],[0.6,0.7]\right), \\
&\left(x_{5},[0.0,0.1],[0.8,0.9]\right),\left(x_{6},[0.7,0.8],[0.1,0.2]\right), \\
&\left(x_{7},[0.5,0.6],[0.3,0.4]\right),\left(x_{8},[0.6,0.7],[0.2,0.3]\right), \\
&\left(x_{9},[1.0,1.0],[0.0,0.0]\right),\left(x_{10},[0.1,0.2],[0.7,0.8]\right), \\
&\left.\left(x_{11},[0.0,0.1],[0.8,0.9]\right),\left(x_{12},[0.7,0.8],[0.1,0.2]\right)\right\},
\end{aligned}
$$$$
A_{3}=\left\{\left(x_{1},[0.4,0.5],[0.3,0.4]\right),\left(x_{2},[0.6,0.7],[0.2,0.3]\right)\right. \text {, }
$$$$
\left(x_{3},[0.9,1.0],[0.0,0.0]\right),\left(x_{4},[0.0,0.1],[0.8,0.9]\right) \text {, }
$$$$
\left(x_{5},[0.0,0.1],[0.8,0.9]\right),\left(x_{6},[0.6,0.7],[0.2,0.3]\right) \text {, }
$$$$
\left(x_{7},[0.1,0.2],[0.7,0.8]\right),\left(x_{8},[0.2,0.3],[0.6,0.7]\right) \text {, }
$$$$
\left(x_{9},[0.5,0.6],[0.2,0.4]\right),\left(x_{10},[1.0,1.0],[0.0,0.0]\right) \text {, }
$$$$
\left.\left(x_{11},[0.3,0.4],[0.4,0.5]\right),\left(x_{12},[0.0,0.1],[0.8,0.9]\right)\right\},
$$$$
A_{4}=\left\{\left(x_{1},[1.0,1.0],[0.0,0.0]\right),\left(x_{2},[1.0,1.0],[0.0,0.0]\right),\right.
$$$$
\left(x_{3},[0.8,0.9],[0.0,0.1]\right),\left(x_{4},[0.7,0.8],[0.1,0.2]\right),
$$$$
\left(x_{5},[0.0,0.1],[0.7,0.9]\right),\left(x_{6},[0.0,0.1],[0.8,0.9]\right) \text {, }
$$$$
\left(x_{7},[0.1,0.2],[0.7,0.8]\right),\left(x_{8},[0.1,0.2],[0.7,0.8]\right) \text {, }
$$$$
\left(x_{9},[0.4,0.5],[0.3,0.4]\right),\left(x_{10},[1.0,1.0],[0.0,0.0]\right) \text {, }
$$$$
\left.\left(x_{11},[0.3,0.4],[0.4,0.5]\right),\left(x_{12},[0.0,0.1][0.8,0.9]\right)\right\},
$$ 


$$
\begin{aligned}
B=\{ & \left(x_{1},[0.9,1.0],[0.0,0.0]\right),\left(x_{2},[0.9,1.0],[0.0,0.0]\right), \\
& \left(x_{3},[0.7,0.8],[0.1,0.2]\right),\left(x_{4},[0.6,0.7],[0.1,0.2]\right), \\
& \left(x_{5},[0.0,0.1],[0.8,0.9]\right),\left(x_{6},[0.1,0.2],[0.7,0.8]\right), \\
& \left(x_{7},[0.1,0.2],[0.7,0.8]\right),\left(x_{8},[0.1,0.2],[0.7,0.8]\right), \\
& \left(x_{9},[0.4,0.5],[0.3,0.4]\right),\left(x_{10},[1.0,1.0],[0.0,0.0]\right), \\
& \left.\left(x_{11},[0.3,0.4],[0.4,0.5]\right),\left(x_{12},[0.0,0.1],[0.7,0.9]\right)\right\} .
\end{aligned}
$$

We have to recognize which class the unknown pattern $B$ belongs to. According to the recognition principle of maximum similarity degree between IVIFSs, the process of recognizing $A_{j}$ to $B$ is derived based on $j=\operatorname{argmax}\left\{S\left(A_{i}, B\right)\right\}$. Using the new similarity measure proposed in this paper and $S_{1}$, we can obtain

$$
\begin{array}{rr}
S\left(A_{1}, B\right)=0.454, & S\left(A_{2}, B\right)=0.4157 \\
S\left(A_{3}, B\right)=0.7456, & S\left(A_{4}, B\right)=0.9594 . \\
S_{1}\left(A_{1}, B\right)=0.4811, & S_{1}\left(A_{2}, B\right)=0.4272 \\
S_{1}\left(A_{3}, B\right)=0.7616, & S_{1}\left(A_{4}, B\right)=0.9563 .
\end{array}
$$

It is obvious that the similarity degrees $S\left(A_{4}, B\right), S_{1}\left(A_{4}, B\right)$ are the largest one in the four different similarity methods and so $B$ belongs to $A_{4}$. So we can know that the quality of the building material is excellent. It can be very useful and easy for us to detect the quality of building material with the help of the similarity measure.

4.2. Multicriteria Group Decision Making. For a decision making problem, let $A=\left\{a_{1}, a_{2}, \ldots, a_{m}\right\}$ be a set of alternatives, let $C=\left\{c_{1}, c_{2}, \ldots, c_{n}\right\}$ be a set of criteria, let $W=$ $\left\{w_{1}, w_{2}, \ldots, w_{n}\right\}$ be a set of criteria weights, and let $D W=$ $\left\{d w_{1}, d w_{2}, \ldots, d w_{l}\right\}$ be a set of decision maker's weights. We also have the condition that $\sum_{i=1}^{n} w_{i}=1, w_{i} \in[0,1]$ and $\sum_{i=1}^{l} d w_{i}=1, d w_{i} \in[0,1]$.

The decision making procedure designed to find the best alternative is given by the following steps.

Step 1. The evaluation of the alternative $a_{i}$ with respect to the criterion $c_{j}$ is an intuitionistic fuzzy number represented by $a_{i j}=\left\langle c_{j},\left[u_{i j}^{-}, u_{i j}^{+}\right],\left[v_{i j}^{-}, v_{i j}^{+}\right]\right\rangle$. In this case, the alternative $a_{i}$ is presented by the following IVIFS:

$$
a_{i}=\left\{\left\langle c_{j},\left[u_{i j}^{-}, u_{i j}^{+}\right],\left[v_{i j}^{-}, v_{i j}^{+}\right]\right\rangle \mid c_{j} \in C\right\},
$$

where $0 \leq u_{i j}^{+}+v_{i j}^{+} \leq 1,0 \leq u_{i j}^{-} \leq u_{i j}^{+} \leq 1,0 \leq v_{i j}^{-} \leq v_{i j}^{+} \leq 1$, $j=1,2, \ldots, n, i=1,2, \ldots, m$.

Step 2. We define an ideal IVIFS for each criterion in the ideal alternative $a^{*}$ as $a_{i j}=\left\langle c_{j},[1,1],[0,0]\right\rangle$ for "excellence." Then, by considering criteria weights and applying formula (3), we can gain the weighted similarity measure between the ideal alternative $a^{*}$ and alternative $a_{i}(i=1,2, \ldots, m)$ for each decision maker:

$$
W S_{k}\left(a_{i}, a^{*}\right)=\sum_{j=1}^{n} \frac{2-\min \left\{\mu_{i}^{-}, v_{i}^{-}\right\}-\min \left\{\mu_{i}^{+}, v_{i}^{+}\right\}}{2+\max \left\{\mu_{i}^{-}, v_{i}^{-}\right\}+\max \left\{\mu_{i}^{+}, v_{i}^{+}\right\}}
$$

Step 3. Calculate the last value of alternatives considering each decision maker's evaluation

$$
W S\left(a_{i}, a^{*}\right)=\sum_{k=1}^{l} W S_{k}\left(a_{i}, a^{*}\right) .
$$

Step 4. Determine the order of alternatives. The larger the value of $W S\left(a_{i}, a^{*}\right)$ is, the better the alternative is.

We compare it with the ideal point method [35] based on interval-valued intuitionistic fuzzy sets used in the multicriteria group decision making. As the ideal point method [35] fits multiperiod and one decision maker, we should improve the method to solve our decision problem which is just one period and multidecision maker. The procedure of ideal point method is as follows.

Step 1. Utilize the UDIFWA operator: $r_{i j}=\left(\left[u_{i j}^{-}, u_{i j}^{+}\right],\left[v_{i j}^{-}, v_{i j}^{+}\right]\right.$, $\left.\left[1-u_{i j}^{+}-v_{i j}^{+}, 1-u_{i j}^{-}-v_{i j}^{-}\right]\right)$.

Step 2. Define $a^{+}$and $a^{-}$as the uncertain intuitionistic fuzzy ideal solution and the uncertain intuitionistic fuzzy negative ideal solution, respectively.

Step 3. Calculate the distance between the alternative $a_{i}$ and $a^{+}, a^{-}$:

$$
\begin{aligned}
& d\left(r_{i}, a^{+}\right)=\sum_{k=1}^{l} d w_{k} \sum_{j=1}^{m} w_{j} d\left(r_{i j}, a^{+}\right), \\
& d\left(r_{i}, a^{-}\right)=\sum_{k=1}^{l} d w_{k} \sum_{j=1}^{m} w_{j} d\left(r_{i j}, a^{-}\right) .
\end{aligned}
$$

Step 4. Calculate the closeness coefficient of each alternative:

$$
c\left(a_{i}\right)=\frac{d\left(r_{i}, a^{-}\right)}{d\left(r_{i}, a^{-}\right)+d\left(r_{i}, a^{+}\right)} .
$$

Step 5. Choose the alternative $a_{i}$ which has the largest values of closeness coefficient. The greater the value $\left(a_{i}\right)$, the better the alternative $a_{i}$.

Example 17 (see [17]). We consider an investment company, which wants to invest money in the best option. There is a panel with four possible alternatives to invest the money: (1) $a_{1}$ is a car company; (2) $a_{2}$ is a food company; (3) $a_{3}$ is a computer company; (4) $a_{4}$ is an arms company. The investment company must consider the following three criteria in order to make decision: (1) $c_{1}$ is the risk analysis; (2) $c_{2}$ is the growth analysis; (3) $c_{3}$ is the environmental impact analysis. The criteria are independent and the criteria weights comprise a vector $W=[0.5,0.3,0.2]^{\prime}$. The four 
TABLE 1: The evaluation of the decision maker.

\begin{tabular}{lllr}
\hline & $c_{1}$ & $c_{2}$ & $c_{3}$ \\
\hline$a_{1}$ & $([0.4,0.5],[0.3,0.4])$ & $([0.4,0.6],[0.2,0.4])$ & $([0.1,0.3],[0.5,0.6])$ \\
$a_{2}$ & $([0.6,0.7],[0.2,0.3])$ & $([0.6,0.7],[0.2,0.3])$ & $([0.4,0.7],[0.1,0.2])$ \\
$a_{3}$ & $([0.3,0.6],[0.3,0.4])$ & $([0.5,0.6],[0.3,0.4])$ & $([0.5,0.6],[0.1,0.3])$ \\
$a_{4}$ & $([0.7,0.8],[0.1,0.2])$ & $([0.6,0.7],[0.1,0.3])$ & $([0.3,0.4],[0.1,0.2])$ \\
\hline
\end{tabular}

possible alternatives $a_{i}(i=1,2,3,4)$ are to be evaluated using the interval-valued intuitionistic fuzzy information by the decision maker under the three criteria (see Table 1).

Then we give the ideal IVIFS for each criterion in the ideal alternative $a^{*}$ as $a^{*}=\left\{\left(c_{1},[1,1],[0,0]\right),\left(c_{2},[1,1]\right.\right.$, $\left.[0,0]),\left(c_{3},[1,1],[0,0]\right)\right\}$. With formula $(30)$, we can get the weighted similarity degree between the ideal alternative $a^{*}$ and alternative $a_{i}(i=1,2,3,4)$ as follows:

$$
\begin{aligned}
& W S\left(a_{1}, a^{*}\right)=0.4418, \quad W S\left(a_{2}, a^{*}\right)=0.6040 \\
& W S\left(a_{3}, a^{*}\right)=0.4997, \quad W S\left(a_{4}, a^{*}\right)=0.6613 .
\end{aligned}
$$

Besides, $k=1$; that is, there is only one decision maker, so we can see that

$$
W S\left(a_{4}, a^{*}\right)>W S\left(a_{2}, a^{*}\right)>W S\left(a_{3}, a^{*}\right)>W S\left(a_{1}, a^{*}\right) .
$$

The order of the alternatives is $a_{4}>a_{2}>a_{3}>a_{1}$. So we can get that the best alternative is $a_{4}$.

Using the ideal point method, we can obtain

$$
\begin{aligned}
& r_{1}=\{([0.4,0.5],[0.3,0.4],[0.1,0.3]) \text {, } \\
& ([0.4,0.6],[0.2,0.4],[0,0.4]), \\
& ([0.1,0.3],[0.5,0.6],[0.1,0.4])\}, \\
& r_{2}=\{([0.6,0.7],[0.2,0.3],[0,0.2]) \text {, } \\
& ([0.6,0.7],[0.2,0.3],[0,0.2]) \text {, } \\
& ([0.4,0.7],[0.1,0.2],[0.1,0.4])\} \text {, } \\
& r_{3}=\{([0.3,0.6],[0.3,0.4],[0,0.4]) \text {, } \\
& ([0.5,0.6],[0.3,0.4],[0,0.2]) \text {, } \\
& ([0.5,0.6],[0.1,0.3],[0.1,0.4])\}, \\
& r_{4}=\{([0.7,0.8],[0.1,0.2],[0,0.2]) \text {, } \\
& ([0.6,0.7],[0.1,0.3],[0,0.3]) \text {, } \\
& ([0.3,0.4],[0.1,0.2],[0.4,0.6])\}, \\
& a^{+}=\{([1,1],[0,0],[0,0]),([1,1],[0,0],[0,0]), \\
& ([1,1],[0,0],[0,0])\}, \\
& a^{-}=\{([0,0],[1,1],[0,0]),([0,0],[1,1],[0,0]), \\
& ([0,0],[1,1],[0,0])\} \text {. }
\end{aligned}
$$

TABLE 2: The results of ideal point method.

\begin{tabular}{lllcl}
\hline & $i=1$ & $i=2$ & $i=3$ & $i=4$ \\
\hline$d\left(r_{i}, a^{-}\right)$ & 0.625 & 0.745 & 0.68 & 0.835 \\
$d\left(r_{i}, a^{+}\right)$ & 0.585 & 0.365 & 0.5 & 0.335 \\
$c\left(a_{1}\right)$ & 0.517 & 0.671 & 0.576 & 0.714 \\
\hline
\end{tabular}

The results of the ideal point method are shown in Table 2. We can know that the results from the ideal point method are also the same as our measures.

Example 18 (see [8]). In order to strengthen academic education and promote the quality of teaching, the school of management of a Chinese university wants to introduce oversea outstanding professor. The panel of the decision makers consist of the university president $d w_{1}$, dean of the management school $d w_{2}$, and human resource officer $d w_{3}$. Besides, the weight of the decision maker is $D W=$ $\{0.5,0.3,0.2\}$. They make strict evaluation for five candidates $a_{i}(i=1,2,3,4,5)$ from four aspects, namely, morality $c_{1}$, research capability $c_{2}$, teaching skills $c_{3}$, and education background $c_{4}$. The weights of the evaluation criteria comprise a vector $W=[0.4,0.3,0.2,0.1]^{\prime}$. The evaluations of each decision maker to the candidates construct the following interval-valued intuitionistic fuzzy information as present in Tables 3, 4, and 5 .

Step 1. In order to calculate the similarity degree between the ideal alternative and the alternative $a_{i}(i=1,2,3,4)$, we have the ideal alternative $a^{*}$ as follows:

$$
\begin{aligned}
a^{*}=\left\{\left(c_{1},[1,1],[0,0]\right),\left(c_{2},[1,1],[0,0]\right),\right. \\
\\
\left.\left(c_{3},[1,1],[0,0]\right),\left(c_{4},[1,1],[0,0]\right)\right\} .
\end{aligned}
$$

It is done by formula (64). The result is shown in Table 6.

Step 2. The last value of each alternative can be calculated by formula (65). Consider

$$
\begin{gathered}
\text { WS }\left(a_{1}, a^{*}\right)=0.5244, \quad W S\left(a_{2}, a^{*}\right)=0.5603, \\
W S\left(a_{3}, a^{*}\right)=0.4662, \quad W S\left(a_{4}, a^{*}\right)=0.5490, \\
W S\left(a_{5}, a^{*}\right)=0.5361 .
\end{gathered}
$$

Step 3. The ranking order of all alternatives is shown as follows:

$$
a_{2}>a_{4}>a_{5}>a_{1}>a_{3} .
$$

Thus, the best alternative is $a_{2}$. 
TABLE 3: The evaluation of the university president.

\begin{tabular}{llccc}
\hline & $c_{1}$ & $c_{2}$ & $c_{3}$ & $c_{4}$ \\
\hline$a_{1}$ & $([0.6,0.8],[0.1,0.2])$ & $([0.2,0.4],[0.4,0.5])$ & $([0.6,0.7],[0.2,0.3])$ & $([0.4,0.5],[0.2,0.4])$ \\
$a_{2}$ & $([0.4,0.7],[0.0,0.1])$ & $([0.5,0.7],[0.1,0.2])$ & $([0.7,0.8],[0.1,0.2])$ & $([0.7,0.8],[0.1,0.2])$ \\
$a_{3}$ & $([0.3,0.7],[0.2,0.3])$ & $([0.2,0.4],[0.4,0.5])$ & $([0.1,0.4],[0.4,0.5])$ & $([0.3,0.4],[0.4,0.6])$ \\
$a_{4}$ & $([0.7,0.8],[0.1,0.2])$ & $([0.2,0.3],[0.4,0.6])$ & $([0.6,0.8],[0.0,0.2])$ & $([0.6,0.8],[0.0,0.2])$ \\
$a_{5}$ & $([0.5,0.6],[0.3,0.4])$ & $([0.7,0.8],[0.0,0.1])$ & $([0.2,0.4],[0.4,0.5])$ & $([0.1,0.3],[0.4,0.6])$ \\
\hline
\end{tabular}

TABLE 4: The evaluation of the dean of the management school.

\begin{tabular}{llcrr}
\hline & $c_{1}$ & $c_{2}$ & $c_{3}$ & $c_{4}$ \\
\hline$a_{1}$ & $([0.2,0.4],[0.4,0.5])$ & $([0.6,0.7],[0.1,0.2])$ & $([0.5,0.7],[0.1,0.2])$ & $([0.5,0.7],[0.1,0.2])$ \\
$a_{2}$ & $([0.6,0.8],[0.0,0.2])$ & $([0.2,0.3],[0.4,0.5])$ & $([0.7,0.8],[0.1,0.2])$ & $([0.2,0.4],[0.4,0.5])$ \\
$a_{3}$ & $([0.1,0.4],[0.4,0.5])$ & $([0.8,0.9],[0.0,0.1])$ & $([0.1,0.4],[0.2,0.5])$ & $([0.4,0.7],[0.2,0.3])$ \\
$a_{4}$ & $([0.6,0.8],[0.0,0.2])$ & $([0.3,0.8],[0.0,0.1])$ & $([0.2,0.3],[0.4,0.6])$ & $([0.6,0.7],[0.2,0.3])$ \\
$a_{5}$ & $([0.2,0.4],[0.5,0.6])$ & $([0.6,0.7],[0.2,0.3])$ & $([0.6,0.8],[0.0,0.2])$ & $([0.1,0.4],[0.3,0.5])$ \\
\hline
\end{tabular}

TABLE 5: The evaluation of the human resource officer.

\begin{tabular}{lcccc}
\hline & $c_{1}$ & $c_{2}$ & $c_{3}$ & $c_{4}$ \\
\hline$a_{1}$ & $([0.2,0.4],[0.4,0.5])$ & $([0.2,0.4],[0.4,0.5])$ & $([0.4,0.7],[0.0,0.1])$ & $([0.7,0.9],[0.0,0.1])$ \\
$a_{2}$ & $([0.2,0.3],[0.4,0.6])$ & $([0.2,0.3],[0.4,0.6])$ & $([0.6,0.7],[0.2,0.3])$ & $([0.5,0.7],[0.1,0.2])$ \\
$a_{3}$ & $([0.8,0.9],[0.0,0.1])$ & $([0.3,0.4],[0.4,0.5])$ & $([0.1,0.3],[0.3,0.5])$ & $([0.2,0.4],[0.4,0.5])$ \\
$a_{4}$ & $([0.3,0.8],[0.1,0.2])$ & $([0.1,0.2],[0.4,0.6])$ & $([0.2,0.3],[0.4,0.5])$ & $([0.3,0.4],[0.4,0.6])$ \\
$a_{5}$ & $([0.7,0.8],[0.0,0.2])$ & $([0.3,0.8],[0.0,0.1])$ & $([0.4,0.7],[0.2,0.3])$ & $([0.6,0.8],[0.1,0.2])$ \\
\hline
\end{tabular}

TABLE 6: The similarity degree results.

\begin{tabular}{|c|c|c|c|c|c|}
\hline & $W S_{k}\left(a_{1}, a^{*}\right)$ & $W S_{k}\left(a_{2}, a^{*}\right)$ & $W S_{k}\left(a_{3}, a^{*}\right)$ & $W S_{k}\left(a_{4}, a^{*}\right)$ & $W S_{k}\left(a_{5}, a^{*}\right)$ \\
\hline$k=1$ & 0.5448 & 0.6449 & 0.416 & 0.5844 & 0.5202 \\
\hline$k=2$ & 0.5459 & 0.5416 & 0.5129 & 0.5752 & 0.4679 \\
\hline$k=3$ & 0.4413 & 0.377 & 0.5217 & 0.4211 & 0.6779 \\
\hline
\end{tabular}

In the same way, we can also get the result based on the ideal point measure:

$$
\begin{aligned}
a^{+}=\{( & (1,1],[0,0],[0,0]),([1,1],[0,0],[0,0]), \\
& ([1,1],[0,0],[0,0]),([1,1],[0,0],[0,0])\}, \\
a^{-}=\{([0,0],[1,1],[0,0]),([0,0],[1,1],[0,0]), & ([0,0],[1,1],[0,0]),([0,0],[1,1],[0,0])\} .
\end{aligned}
$$

Table 7 describes the results of the ideal point method. We can get the same ranking order and the same best alternative from the two methods. We can conclude that the ideal point method and our similarity measure method have the same effect used to solve the multicriteria group decision making problem. The difference is that the method proposed in [35] uses the distance measure and closeness coefficient measure while our method takes advantage of the similarity measure.

\section{Conclusion}

In this paper, we have presented a new similarity measure for interval-valued intuitionistic fuzzy sets with considering the
TABLE 7: The results of ideal point method.

\begin{tabular}{lccccc}
\hline & $i=1$ & $i=2$ & $i=3$ & $i=4$ & $i=5$ \\
\hline$d\left(r_{i}, a^{-}\right)$ & 0.7435 & 0.824 & 0.705 & 0.7865 & 0.7545 \\
$d\left(r_{i}, a^{+}\right)$ & 0.528 & 0.4805 & 0.597 & 0.4875 & 0.4905 \\
$c\left(a_{1}\right)$ & 0.585 & 0.632 & 0.541 & 0.617 & 0.606 \\
\hline
\end{tabular}

hesitancy degree and using the entropy measure of IVIFSs. In fact, the entropy measure has been used in constructing the similarity measure, such as Wei et al. [24]. However, these similarity measures including many other definition methods $[16-18,33]$ may make mistakes, especially when the differences of membership and nonmembership between the candidate fuzzy sets and the target one are close and the difference of hesitancy degree among the candidate fuzzy sets is large. In order to make up for the flaws, we take the influence of the hesitancy degree into account and give a new method to construct the similarity measure by entropy measure, by which the proposed measure is demonstrated to yield a similarity measure. Then, the efficiency of proposed 
similarity measure is demonstrated by the comparative analysis with the other existing similarity measures. Finally, we also apply the new similarity measure to expert system to solve the pattern recognition problem and the multicriteria group decision making problem in which we compare our method with the ideal point method [35]. Several examples are given to illustrate the practicality and effectiveness of the applications. Besides, the similarity measure between interval-valued intuitionistic fuzzy sets can be applied to many different fields.

\section{Conflict of Interests}

The authors declare that there is no conflict of interests regarding the publication of this paper.

\section{Acknowledgments}

This study was partly funded by National Natural Science Foundation of China (71271070) and China Scholarship Council (201306120159).

\section{References}

[1] K. Atanassov and G. Gargov, "Interval valued intuitionistic fuzzy sets," Fuzzy Sets and Systems, vol. 31, no. 3, pp. 343-349, 1989.

[2] D.-F. Li, "Closeness coefficient based nonlinear programming method for interval-valued intuitionistic fuzzy multiattribute decision making with incomplete preference information," Applied Soft Computing Journal, vol. 11, no. 4, pp. 3402-3418, 2011.

[3] V. Lakshmana Gomathi Nayagam and G. S. Geetha Sivaraman, "Ranking of interval-valued intuitionistic fuzzy sets," Applied Soft Computing Journal, vol. 11, no. 4, pp. 3368-3372, 2011.

[4] G.-W. Wei, H.-J. Wang, and R. Lin, "Application of correlation coefficient to interval-valued intuitionistic fuzzy multiple attribute decision-making with incomplete weight information," Knowledge and Information Systems, vol. 26, no. 2, pp. 337349, 2011.

[5] Q.-S. Zhang, S. Jiang, B. Jia, and S. Luo, "Some information measures for interval-valued intuitionistic fuzzy sets," Information Sciences, vol. 180, no. 24, pp. 5130-5145, 2010.

[6] P. Burillo and H. Bustince, "Entropy on intuitionistic fuzzy sets and on interval-valued fuzzy sets," Fuzzy Sets and Systems, vol. 78, no. 3, pp. 305-316, 1996.

[7] M. H. Fazel Zarandi, B. Rezaee, I. B. Turksen, and E. Neshat, "A type-2 fuzzy rule-based expert system model for stock price analysis," Expert Systems with Applications, vol. 36, no. 1, pp. 139-154, 2009.

[8] Z. H. Zhang, M. Wang, J. Y. Yang, Y. P. Ye, and Y. F. Li, "A dynamic interval-valued intuitionistic fuzzy sets applied to pattern recognition," Mathematical Problems in Engineering, vol. 2013, Article ID 408012, 16 pages, 2013.

[9] Y. J. Zhang, P. H. Li, Y. Z. Wang, P. J. Ma, and X. H. Su, "Multiattribute decision making based on entropy under interval-valued intuitionistic fuzzy environment," Mathematical Problems in Engineering, vol. 2013, Article ID 526871, 8 pages, 2013.

[10] M. H. Fazel Zarandi, B. Rezaee, I. B. Turksen, and E. Neshat, "A type-2 fuzzy rule-based expert system model for stock price analysis," Expert Systems with Applications, vol. 36, no. 1, pp. 139-154, 2009.

[11] A. Kele, A. Kele, and U. Yavuz, "Expert system based on neurofuzzy rules for diagnosis breast cancer," Expert Systems with Applications, vol. 38, no. 5, pp. 5719-5726, 2011.

[12] Y. L. Li, C. Wu, P. Luo, and W. Zhang, "Exploring the characteristics of innovation adoption in social networks: structure, homophily, and strategy," Entropy, vol. 15, pp. 2662-2678, 2013.

[13] S.-M. Chen, L.-W. Lee, H.-C. Liu, and S.-W. Yang, "Multiattribute decision making based on interval-valued intuitionistic fuzzy values," Expert Systems with Applications, vol. 39, pp. 10343-10351, 2012.

[14] L. Y. Zhang, X. H. Xu, and L. Tao, "Some similarity measures for triangular fuzzy number and their applications in multiple criteria group decision-making," Journal of Applied Mathematics, vol. 2013, Article ID 538261, 7 pages, 2013.

[15] Y. L. Li, C. Wu, X. D. Wang, and S. T. Wu, "A tree-network model for mining short message services seed users and its empirical analysis," Knowledge-Based Systems, vol. 40, pp. 50-57, 2013.

[16] J. Ye, "Multicriteria decision-makingmethod using the Dice similarity measure based on the reduct intuitionistic fuzzy sets of interval-valued intuitionistic fuzzy sets," Applied Mathematical Modelling, vol. 36, no. 9, pp. 4466-4472, 2012.

[17] P. Jaccard, "Distribution de la flore alpine dans le Bassin des Drouces et dans quelques regions voisines," Bulletin de la Société Vaudoise des Sciences Naturelles, vol. 37, no. 140, pp. 241-272, 1901.

[18] L. R. Dice, "Measures of the amount of ecologic association between species," Ecology, vol. 26, pp. 297-302, 1945.

[19] G. Salton and M. J. McGill, Introduction to Modern Information Retrieval, McGraw-Hill, New York, NY, USA, 1987.

[20] P. Luo, Y. L. Li, and C. Wu, "A new similarity measure of intervalvalued intuitionistic fuzzy sets and its application in commodity recommendation," International Journal on Information Technology, no. 3, pp. 186-192, 2013.

[21] J. Ye, "Interval-valued intuitionistic fuzzy cosine similarity measures for multiple attribute decision-making," International Journal of General Systems, no. 1, pp. 101-105, 2012.

[22] Z. S. Xu and R. R. Yager, "Intuitionistic and interval-valued intuitionistic fuzzy preference relations and their measures of similarity for the evaluation of agreement within a group," Fuzzy Optimization and Decision Making, vol. 8, no. 2, pp. 123-139, 2009.

[23] Z. S. Xu and J. Chen, "An overview of distance and similarity measures of intuitionistic fuzzy sets," International Journal of Uncertainty, Fuzziness and Knowledge-Based Systems, vol. 16, no. 4, pp. 529-555, 2008.

[24] C.-P. Wei, P. Wang, and Y.-Z. Zhang, "Entropy, similarity measure of interval-valued intuitionistic fuzzy sets and their applications," Information Sciences, vol. 181, no. 19, pp. 42734286, 2011.

[25] P. Luo, Y. L. Li, and C. Wu, "Complex networks evolution research with using the network structure entropy," Complex Systems and Complexity Science, vol. 10, no. 4, pp. 62-68, 2013.

[26] P. Singh, "A new method on measure of similarity between interval-valued intuitionistic fuzzy sets for pattern recognition," Journal of Applied \& Computational Mathematics, vol. 1, no. 1, pp. 1-5, 2012.

[27] Z. Xu and R. R. Yager, "Dynamic intuitionistic fuzzy multiattribute decison making," International Journal of Approximate Reasoning, vol. 48, no. 1, pp. 246-262, 2008. 
[28] Z. Xu, "Some similarity measures of intuitionistic fuzzy sets and their applications to multiple attribute decision making," Fuzzy Optimization and Decision Making, vol. 6, no. 2, pp. 109-121, 2007.

[29] M. Xia and Z. Xu, "Some new similarity measures for intuitionistic fuzzy values and their application in group decision making," Journal of Systems Science and Systems Engineering, vol. 19, no. 4, pp. 430-452, 2010.

[30] Z. Xu and M. Xia, "Distance and similarity measures for hesitant fuzzy sets," Information Sciences, vol. 181, no. 11, pp. 2128-2138, 2011.

[31] Z. S. Xu, "On similarity measures of interval-valued intuitionistic fuzzy sets and their application to pattern recognitions," Journal of Southeast University (English Edition), vol. 23, no. 1, pp. 139-143, 2007.

[32] W.-L. Hung and M.-S. Yang, "Similarity measures of intuitionistic fuzzy sets based on Hausdorff distance," Pattern Recognition Letters, vol. 25, no. 14, pp. 1603-1611, 2004.

[33] S. M. Chen, "Measures of similarity between vague sets," Fuzzy Sets and Systems, vol. 74, no. 2, pp. 217-223, 1995.

[34] P. Singh, "A new method on measure of similarity between interval-valued intuitionistic fuzzy sets for pattern recognition," Journal of Applied \& Computational Mathematics, vol. 1, no. 1, pp. 1-5, 2012.

[35] Z. Xu and R. R. Yager, "Dynamic intuitionistic fuzzy multiattribute decison making," International Journal of Approximate Reasoning, vol. 48, no. 1, pp. 246-262, 2008. 


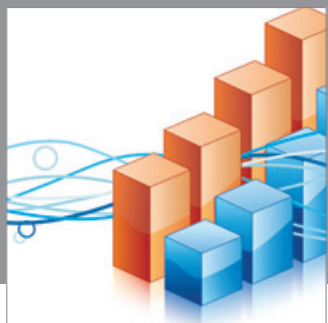

Advances in

Operations Research

mansans

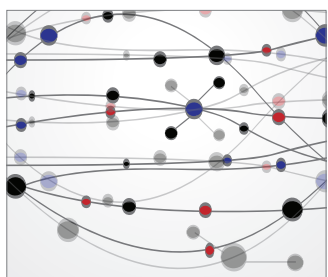

The Scientific World Journal
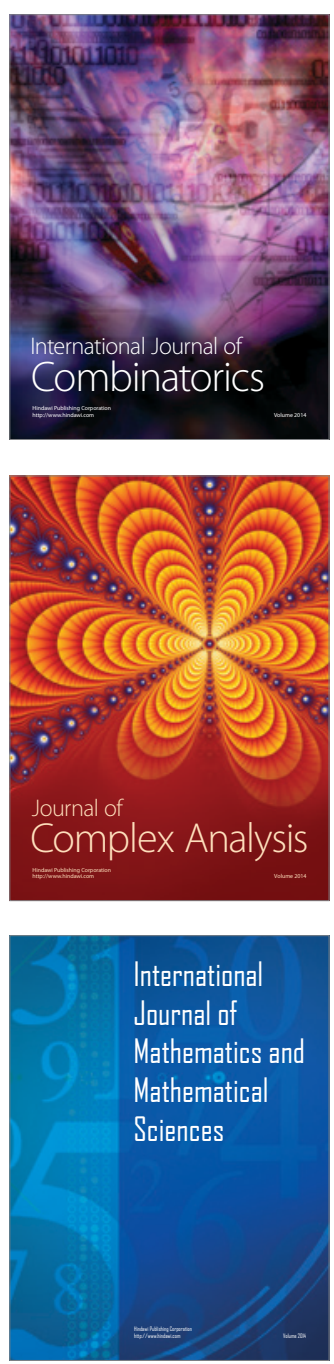
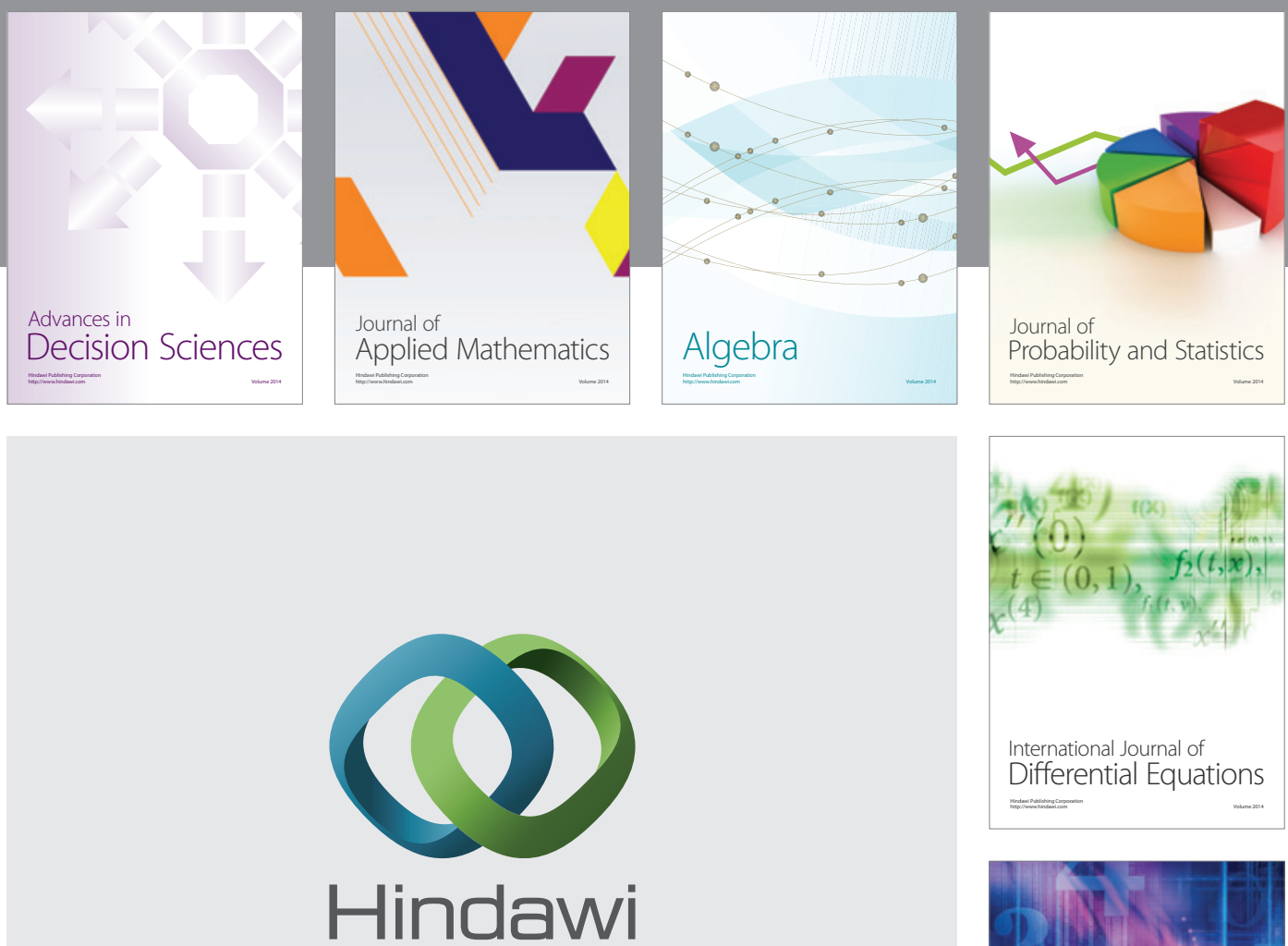

Submit your manuscripts at http://www.hindawi.com
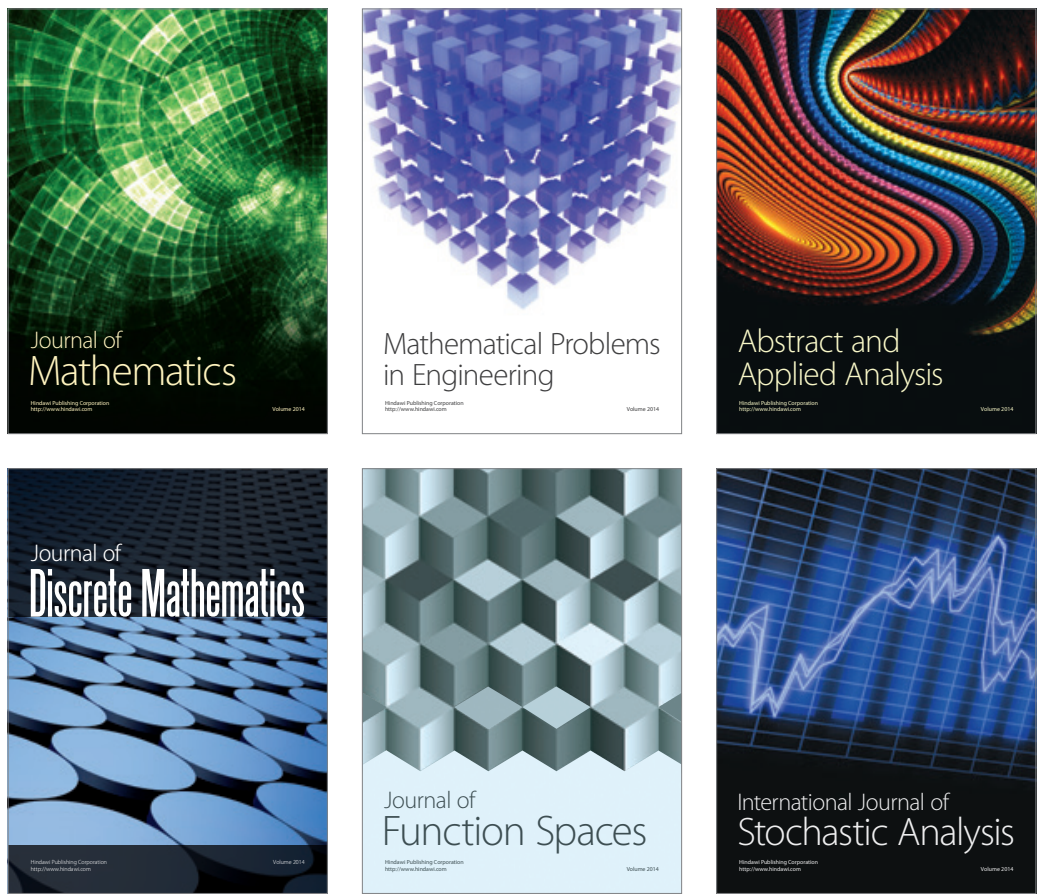

Journal of

Function Spaces

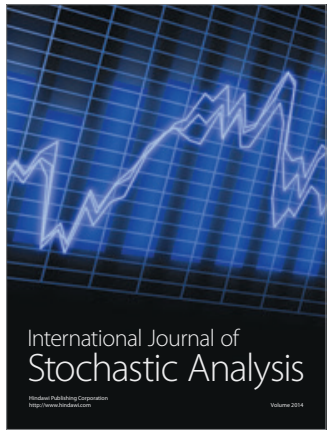

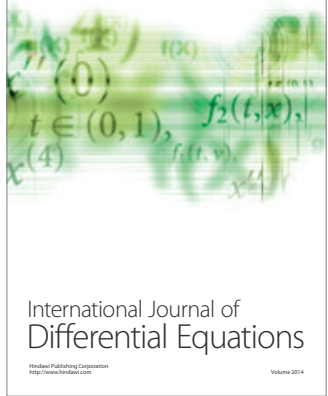
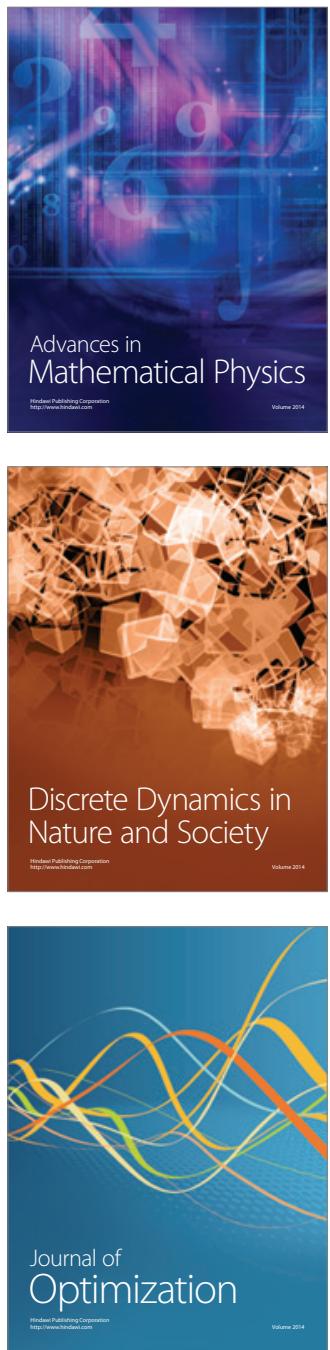